\title{
STELLAR YIELDS FROM METAL-RICH ASYMPTOTIC GIANT BRANCH MODELS
}

\author{
Amanda I. Karakas ${ }^{1,2}$ and Maria Lugaro ${ }^{2,3}$ \\ ${ }^{1}$ Research School of Astronomy and Astrophysics, Australian National University, Canberra, ACT 2611, Australia; amanda.karakas@anu.edu.au \\ ${ }^{2}$ Monash Centre for Astrophysics, School of Physics and Astronomy, Monash University, VIC 3800, Australia \\ ${ }^{3}$ Konkoly Observatory, Research Centre for Astronomy and Earth Sciences, Hungarian Academy of Sciences, H-1121 Budapest, Hungary \\ Received 2016 January 12; revised 2016 March 21; accepted 2016 April 6; published 2016 June 27
}

\begin{abstract}
We present new theoretical stellar yields and surface abundances for three grids of metal-rich asymptotic giant branch (AGB) models. Post-processing nucleosynthesis results are presented for stellar models with initial masses between $1 M_{\odot}$ and $7.5 M_{\odot}$ for $Z=0.007$, and $1 M_{\odot}$ and $8 M_{\odot}$ for $Z=0.014$ (solar) and $Z=0.03$. We include stellar surface abundances as a function of thermal pulse on the AGB for elements from $\mathrm{C}$ to $\mathrm{Bi}$ and for a selection of isotopic ratios for elements up to $\mathrm{Fe}$ and $\mathrm{Ni}$ (e.g., ${ }^{12} \mathrm{C} /{ }^{13} \mathrm{C}$ ), which can be obtained from observations of molecules in stars and from the laboratory analysis of meteoritic stardust grains. Ratios of elemental abundances of $\mathrm{He} / \mathrm{H}, \mathrm{C} / \mathrm{O}$, and $\mathrm{N} / \mathrm{O}$ are also included, which are useful for direct comparison to observations of AGB stars and their progeny, including planetary nebulae. The integrated elemental stellar yields are presented for each model in the grid for hydrogen, helium, and all stable elements from $\mathrm{C}$ to Bi. Yields of Li are also included for intermediatemass models with hot bottom burning. We present the first slow neutron-capture (s-process) yields for super solar metallicity AGB stars with $Z=0.03$, and the first complete $s$-process yields for models more massive than $6 M_{\odot}$ at all three metallicities.
\end{abstract}

Key words: Galaxy: abundances - galaxies: abundances - ISM: abundances - nuclear reactions, nucleosynthesis, abundances - stars: AGB and post-AGB - stars: carbon

Supporting material: FITS files

\section{INTRODUCTION}

Theoretical stellar nucleosynthesis calculations are an important dataset for the interpretation of chemical abundances that are derived from spectra of stars and gaseous regions in galaxies. When the abundances are from samples of old, lowmass stars they allow us to disentangle the processes of galaxy formation and evolution; the study of Galactic archeology (Freeman \& Bland-Hawthorn 2002). In this framework, stellar abundances are compared to theoretical predictions from chemical evolution models, which require as input the chemical yields from stars under the assumption that the yields from previous generations of stars have contributed to the build up of elements over time (Romano et al. 2010; Kobayashi et al. 2011a, 2011b; Shingles et al. 2014; Mollá et al. 2015).

Theoretical nucleosynthesis calculations are also essential for a direct comparison between predicted stellar abundances and observations. This is especially so for evolved stars that are on the red giant branch (RGB) and the asymptotic giant branch (AGB), or have evolved to become post-AGB stars and planetary nebulae (PNs). In this case, comparison between theory and observation can provide insights into our understanding of stellar astrophysics. If we fail to explain the amount of neutron-capture elements on the surface of a post-AGB star, for example, clearly the model needs improving (e.g., De Smedt et al. 2012). Furthermore, detailed stellar nucleosynthesis calculations providing isotopic abundances are needed for comparison to direct observations of isotopic ratios from molecular lines (e.g., Kahane et al. 2000; Lederer \& Aringer 2009; Milam et al. 2009; Fonfría et al. 2015). They also represent the key to interpret the composition of stable and radioactive isotopes in meteoritic components, such as stardust grains (Zinner 2014, pp. 181-213) and calcium-aluminum inclusion (e.g., Akram et al. 2013), as well as meteoritic leachates and whole rocks (e.g., Dauphas et al. 2002; Akram et al. 2015; Burkhardt \& Schönbächler 2015), with implications on the origin of cosmic dust and on the formation of the solar system.

Low- and intermediate-mass stars cover a range in mass from $0.8-8 M_{\odot}$, depending on metallicity (see Figure 1 from Karakas \& Lattanzio 2014). Stars with initial masses in this range will evolve through core hydrogen and helium burning before ascending the AGB (Busso et al. 1999; Herwig 2005; Karakas \& Lattanzio 2014). It is during the AGB phase that the richest nucleosynthesis occurs, driven by He-shell instabilities. These instabilities or thermal pulses (TP) may result in mixing between the $\mathrm{H}$-exhausted core and the envelope; this is known as third dredge up (TDU). The TDU will alter the composition of the envelope by bringing the products of He-shell burning and the elements produced by the slow neutron-capture process (the $s$-process) to the stellar surface.

Low-mass AGB stars with initial masses $M \lesssim 4 M_{\odot}$ have surface compositions and stellar yields characterized by enrichments in carbon, nitrogen, fluorine, and $s$-process elements (e.g., Busso et al. 2001; Karakas et al. 2007; Cristallo et al. 2009; Weiss \& Ferguson 2009). In contrast, intermediatemass AGB stars with initial masses $M \gtrsim 4 M_{\odot}$ experience both the second dredge up (SDU) during the early AGB, which results in large increases in helium and nitrogen, and hot bottom burning (HBB), the process by which the base of the envelope becomes hot enough for proton-capture nucleosynthesis (Karakas \& Lattanzio 2003; Ventura et al. 2013). The surface chemistry of intermediate-mass stars is thus characterized by proton-capture nucleosynthesis, perhaps with some contribution from He-shell burning and the s-process (Karakas et al. 2012).

Karakas \& Lattanzio (2014) reviewed the available stellar yields from AGB models. The most significant gaps were found for low-metallicity AGB models and for the yields of 
$s$-process elements. For metallicities around solar, the only set of tabulated stellar yields of $s$-process elements are those by Cristallo et al. (2015), which are available on the FRUITY online database. ${ }^{4}$ The NuGrid collaboration is also in the process of publishing yields for AGB stars of metallicity around solar $(Z=0.02$ and 0.01$)$ for a limited range of masses (e.g., Pignatari et al. 2013). No models exist for metallicities higher than $Z=0.02$.

Stellar yields are known to be highly uncertain and dependent on the model assumptions used in the stellar evolutionary (and post-processing, if used) calculations (Ventura \& D'Antona 2005a, 2005b; Stancliffe \& Jeffery 2007; Karakas 2010). These uncertainties manifest into uncertainties in chemical evolution studies (Romano et al. 2010). This means that yields by different groups vary as a consequence of assumptions about the treatment of convection and convective borders and the adopted mass-loss rates. For that reason, it is essential to provide yields from different stellar evolution codes in order to understand what elements are most affected by stellar modeling uncertainties.

Comparing yield sets is useful because it can reveal how different choices in the input physics affects the yields. However, it does not provide per se an indication of the reliability of any set of stellar yields. Until we are able to constrain how many TPs an AGB star of a given initial mass and metallicity is expected to go through, the stellar yields will remain uncertain. Independent observational tests are the most reliable method to test the validity of any set of stellar models. Such a comparison will be the focus of follow up studies, where we confront our predictions with available observational data for AGB stars, PNs, and postAGB stars, and pre-solar grains.

In Karakas (2014), we provided stellar evolutionary tracks for an updated set of low- and intermediate-mass stellar models between 1 and $8 M_{\odot}{ }^{5}$ In particular, we included models of solar metallicity $(Z=0.014)$, super solar $(Z=0.03)$, and a factor of two below solar $(Z=0.007)$. Karakas (2014) examined the effect of helium enrichment on the production of carbon stars. It was found that modest $(\Delta Y \approx 0.05-0.1)$ increases in helium abundance above the canonical value inhibits carbon star production. This because less He-intershell material is dredged to the surface, which also means that the stellar yields of other elements (e.g., s-process elements in particular) will be reduced. This has been shown to be the case in low-metallicity AGB models (Karakas et al. 2014; Shingles et al. 2015).

In this study we aim to provide theoretical nucleosynthesis predictions including $s$-process elements from the grid of stellar evolutionary models from Karakas (2014) with a canonical helium composition. For the first time, we include surface abundances and stellar yields for masses up to the $\mathrm{CO}$ core limit $\left(\approx 8 M_{\odot}\right.$, see Section 2$)$ and models of super solar AGB stars of $Z=0.03$. We also aim to provide abundances in a form that is useful to the AGB, post-AGB, and PNs communities to allow for a direct comparison to abundances of these objects.

Section 2 discusses the stellar evolutionary models used as input into our post-processing nucleosynthesis calculations, while our nucleosynthesis results are summarized in Section 3. We finish with a discussion and conclusion.

\footnotetext{
4 FUll-Network Repository of Updated Isotopic Tables and Yields: http:// fruity.oa-teramo.inaf.it/.

5 We did not provide evolutionary tracks for the post-AGB and white dwarf phases, and refer to the recent evolutionary calculations by, e.g., Miller Bertolami (2015)
}

\section{THE STELLAR MODELS}

For the post-processing nucleosynthesis calculations we use as input the stellar evolutionary models described in Karakas (2014). We consider here only the models with a canonical helium composition, which is $Y=0.26$ for $Z=0.007, Y=0.28$ for $Z=0.014$, and $Y=0.30$ for $Z=0.03$. The grids include models of solar metallicity, defined here to be $Z=0.014$ (based on the solar abundances from Asplund et al. 2009), and a factor of two above and below solar: $Z=0.007$ and $Z=0.03$.

While we refer to Karakas (2014) for the full details of the input physics and the numerical method, we remind the reader of the input physics most relevant to the stellar nucleosynthesis. No mass loss is used on the RGB and we refer to Karakas (2014) for a justification of this choice. We use the Vassiliadis \& Wood (1993) mass-loss rate on the AGB phase. We use the mixinglength theory of convection with a mixing-length parameter $\alpha=1.86$ and assume instantaneous mixing in convective regions. No convective overshoot is included in the calculations prior to the AGB. Dealing with the borders between radiative and convective regions in stellar interiors is a major uncertainty. We implement an algorithm to try to search for a neutrally stable point from the formal Schwarzschild boundary as described by Lattanzio (1986). This method has been shown to increase the efficiency of TDU, at least in intermediate-mass models of $\approx 5 M_{\odot}$ (Frost \& Lattanzio 1996), but not in lower mass models close to the minimum mass for carbon stars (Kamath et al. 2012).

The models cover the complete range of AGB masses from $1 M_{\odot}$ to the upper limit for producing a $\mathrm{CO}$ core, which is $8 M_{\odot}$ for $Z=0.014$ and $Z=0.03$ and $7 M_{\odot}$ for $Z=0.007$. The $8 M_{\odot}$ models (and the $7 M_{\odot}$ for $Z=0.007$ ) produce a hybrid $\mathrm{CO}(\mathrm{Ne})$ core and experience off-center carbon flashes, but the temperature in the core is not high enough to ignite a carbon flame that reaches the center (e.g., as described by Siess 2006). Above these masses, stars will become ONe super-AGB stars or neutron stars (Doherty et al. 2015).

We supplement the calculations in Karakas (2014) with extra stellar evolutionary model calculations such that we have a grid of models with a mass spacing of $\Delta M=0.25 M_{\odot}$ up to $5 M_{\odot}$; above that mass we assume the same grid as described in Karakas (2014). The new models have masses $M=2.75,3.25$, $3.75,4.25$, and $4.75 M_{\odot}$ for the metallicities where we did not provide these masses before. We also include a $7.5 M_{\odot}$, $Z=0.007$ model, which evolves through complete core $\mathrm{C}$ burning before ascending the AGB as a ONe-core, super-AGB star (e.g., Doherty et al. 2014). This is to make sure that we have a fine enough mass grid such that we do not miss any important nonlinear behavior in the stellar yields. The same stellar evolutionary code and input physics were adopted for the new calculations for consistency.

The theoretical minimum initial mass for producing a solar metallicity, carbon-rich star in Karakas (2014) is $2 M_{\odot}$. This is likely above the observational limit of $\approx 1.5 M_{\odot}$, which is derived directly from observations of C-stars in binary systems and open clusters (Groenewegen et al. 1995), although uncertainties are large and statistics are low. A mass of $\approx 1.5 M_{\odot}$ is also derived by comparison of theoretical models to observationally derived carbon-star luminosity functions in the Galaxy and Magellanic Clouds (e.g., Groenewegen et al. 1995; Marigo et al. 1999; Stancliffe et al. 2005; Cristallo et al. 2011). Groenewegen et al. (1995) estimate the minimum mass for solar metallicity C-stars to lie between $1.5-1.6 M_{\odot}$, where their $Z_{\text {solar }}=0.02$. Using updated observational data, Cristallo et al. 
Table 1

Stellar Models Calculated in Addition to those in Karakas (2014)

\begin{tabular}{|c|c|c|c|c|c|c|c|c|c|c|c|}
\hline $\begin{array}{l}\text { Mass } \\
\left(M_{\odot}\right)\end{array}$ & SDU & HBB & TDU & $\# \mathrm{TP}$ & $\lambda_{\max }$ & $\begin{array}{c}M_{\mathrm{c}}(1) \\
\left(M_{\odot}\right)\end{array}$ & $\begin{array}{l}T_{\mathrm{bce}}^{\max } \\
(\mathrm{MK})\end{array}$ & $\begin{array}{c}L_{\mathrm{agb}}^{\max } \\
\left(L_{\odot}\right)\end{array}$ & $\begin{array}{c}\tau_{\text {stellar }} \\
(\mathrm{Myr})\end{array}$ & $\begin{array}{c}\tau_{\mathrm{agb}} \\
(\mathrm{Myr})\end{array}$ & $\begin{array}{l}\tau_{\text {tpagb }} \\
(\mathrm{Myr})\end{array}$ \\
\hline \multicolumn{12}{|c|}{$Z=0.007, Y=0.26$ models. } \\
\hline 1.50 & No & No & Yes $^{\mathrm{a}}$ & 18 & 0.31 & 0.548 & 3.77 & $8.39(3)$ & 2451 & 18.67 & 2.057 \\
\hline 1.75 & No & No & Yes $^{\mathrm{a}}$ & 17 & 0.51 & 0.556 & 4.13 & $8.68(3)$ & 1535 & 17.05 & 1.836 \\
\hline 2.75 & No & No & Yes & 25 & 0.80 & 0.589 & 5.11 & $1.22(4)$ & 509.6 & 13.09 & 2.086 \\
\hline 3.25 & No & No & Yes & 22 & 0.92 & 0.698 & 11.3 & $1.54(4)$ & 313.4 & 6.115 & 0.920 \\
\hline 3.75 & No & No & Yes & 22 & 0.97 & 0.786 & 26.8 & $1.94(4)$ & 212.2 & 3.964 & 0.503 \\
\hline 4.75 & Yes & Yes & Yes & 55 & 0.95 & 0.865 & 80.5 & $3.43(4)$ & 118.0 & 2.076 & 0.528 \\
\hline 7.50 & Yes & Yes & Yes & 87 & 0.83 & 1.107 & 109 & $7.32(4)$ & 42.6 & 0.383 & 0.075 \\
\hline \multicolumn{12}{|c|}{$Z=0.014, Y=0.28$ models. } \\
\hline 1.50 & No & No & Yes $^{b}$ & 16 & 0.51 & 0.552 & 6.24 & $7.24(3)$ & 2882 & 18.26 & 1.512 \\
\hline 1.75 & No & No & $\mathrm{Yes}^{\mathrm{c}}$ & 20 & 0.50 & 0.554 & 5.43 & $8.73(3)$ & 1755 & 18.61 & 1.736 \\
\hline 3.75 & No & No & Yes & 23 & 0.95 & 0.740 & 18.7 & $3.10(4)$ & 229.4 & 5.129 & 0.620 \\
\hline 4.25 & Yes & Yes & Yes & 31 & 0.96 & 0.840 & 61.9 & $2.67(4)$ & 162.9 & 3.176 & 0.377 \\
\hline 4.75 & Yes & Yes & Yes & 35 & 0.95 & 0.855 & 68.8 & $2.90(4)$ & 121.9 & 2.322 & 0.339 \\
\hline \multicolumn{12}{|c|}{$Z=0.03, Y=0.30$ models. } \\
\hline 2.50 & No & No & Yes $^{d}$ & 30 & 0.81 & 0.547 & 8.82 & $1.08(4)$ & 914.8 & 26.44 & 2.211 \\
\hline 2.75 & No & No & Yes $^{c}$ & 33 & 0.80 & 0.559 & 9.03 & $1.21(4)$ & 695.3 & 21.17 & 2.076 \\
\hline 3.00 & No & No & Yes $^{\mathrm{a}}$ & 33 & 0.81 & 0.580 & 8.24 & $1.32(4)$ & 532.4 & 16.90 & 1.719 \\
\hline
\end{tabular}

Notes. The luminosity is in the format $n(m)$ where $=n \times 10^{m} L_{\odot}$.

${ }^{\text {a }} N_{\mathrm{ov}}=1$; see the text for details.

${ }^{\mathrm{b}} N_{\mathrm{ov}}=3$.

c $N_{\mathrm{ov}}=2$.

d $N_{\mathrm{ov}}=2.5$.

(2011) provide a minimum mass as a function of metallicity: for $Z=0.02$ the minimum mass is $1.5 M_{\odot}$ and for $Z=0.01$ the minimum mass is $1.4 M_{\odot}$.

It should be stressed that the minimum mass derived from these studies is dependent upon the underlying assumptions in the theoretical calculations as well as the uncertainties in the photometry. This can be highlighted by examining the results from the study by Kalirai et al. (2014), who use white dwarfs in open clusters to study the core mass growth and initial-final mass relation. These authors come to conclusion that no TDU takes place in stars less than $2 M_{\odot}$ for a metallicity $Z=0.02$ (which they assume is slightly super solar). The Kalirai et al. (2014) results are also model-dependent but depend on a different code (Marigo et al. 2013). GAIA will provide much help here by providing the distances and hence luminosities to well known bright $\mathrm{C}$-rich stars in the Galaxy. Until then, the minimum mass for $\mathrm{C}$-stars in the Galaxy is not accurately known and probably lies somewhere between 1.4 and $2 M_{\odot}$.

Motivated by these uncertainties, we include convective overshoot at the base of the convective envelope such that a selection of low-mass AGB models also become C-rich. These are the $M=1.5,1.75 M_{\odot}, Z=0.007,0.014$ models, and the $M=2.5$, $2.75,3 M_{\odot}, Z=0.03$ models. For the $Z=0.03$ models we do not have observational clues as to the minimum mass for C-stars but it is likely $\gtrsim 2 M_{\odot}$ (Kalirai et al. 2014). Given the uncertainty in the minimum mass for C-stars in the Galaxy, we provide surface abundances and yields from both calculations: those with overshoot and those without. The prescription we use to include overshoot is the same as used by Karakas et al. (2010) and Kamath et al. (2012), and extends the base of the envelope by $N_{\text {ov }}$ pressure scale heights during dredge up. In order for the masses considered here to become C-rich, we use $N_{\text {ov }} \leqslant 3$, with the specific values used given in the footnotes of Table 1 . These values are consistent with what was found by Kamath et al. (2012) in order to reproduce the observed $\mathrm{M} / \mathrm{C}$ transition luminosity of AGB stars in three Magellanic Cloud clusters. This convective overshoot prescription is not applied to models above $1.75 M_{\odot}$ for $Z \leqslant 0.014$ and above $3 M_{\odot}$ for $Z=0.03$ so does not affect intermediate-mass models with $\mathrm{HBB}$.

Table 1 shows some the relevant properties of the additional AGB models calculated, including the initial stellar mass, whether the models experience SDU, HBB, and TDU. For the cases where we include overshoot, we provide a footnote with the $N_{\text {ov }}$ parameter used. We include the total number of TPs (\#TP), the maximum value of the dredge up efficiency parameter, ${ }^{6} \lambda_{\max }$, the core mass at the first TP, $M_{\mathrm{c}}(1)$, the maximum temperature at the base of the convective envelope, $T_{\mathrm{bce}}^{\max }$, and the maximum surface luminosity during the AGB, $L_{\mathrm{agb}}^{\max }$. For low-mass AGB stars $\lesssim 4 M_{\odot}$, the maximum luminosity occurs at the tip of the AGB and is generally higher than the luminosity when the star becomes C-rich (Kamath et al. 2012). For models with HBB, the maximum luminosity occurs before the tip of the AGB when $\mathrm{HBB}$ is active. We also provide the total stellar lifetime, $\tau_{\text {stellar, }}$, the AGB lifetime, $\tau_{\mathrm{agb}}$, and the thermally pulsing AGB lifetime, $\tau_{\text {tpagb. }}$. Units are megayears for lifetimes, solar units for masses and luminosities, and $\mathrm{MK}\left(10^{6} \mathrm{~K}\right)$ for temperatures.

In Figure 1 we show the total amount of mass dredged into the envelope by TDU, $M_{\text {dredge. }}$ In this figure and from now on, unless specified, we present and discuss models with convective

\footnotetext{
$6 \lambda=\Delta M_{\text {dredge }} / \Delta M_{\mathrm{c}}$, where $\Delta M_{\text {dredge }}$ is the amount of material dredged up, and $\Delta M_{\mathrm{c}}$ is the core mass growth during the preceding interpulse phase; see Karakas et al. (2002).
} 


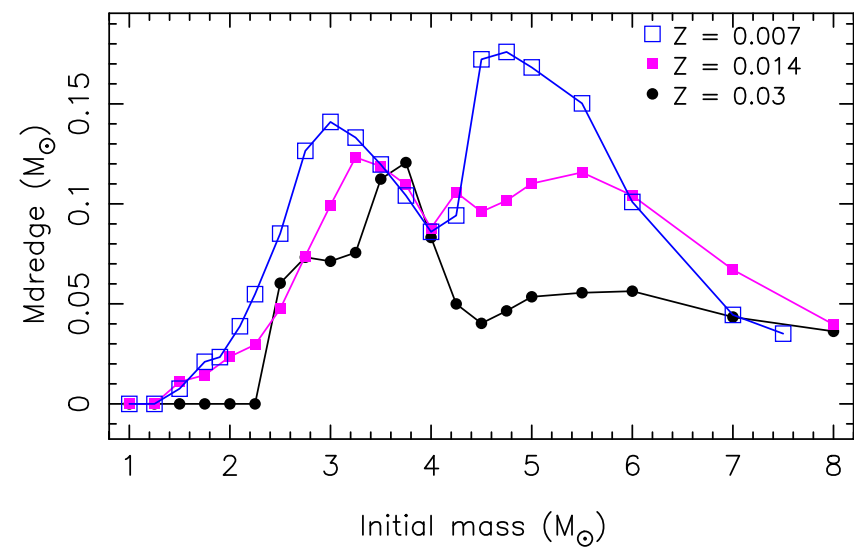

Figure 1. Total amount of mass dredged up to the envelope by TDU for models of $Z=0.007, Z=0.014$, and $Z=0.03$.

overshoot that become C-rich (e.g., the models from Table 1) in preference to models of the same mass without overshoot. Figure 1 shows that the amount of material dredged up increases with decreasing metallicity for a given mass. The behavior of $M_{\text {dredge }}$ with initial stellar mass is interesting: the most massive models above $7 M_{\odot}$ show smaller values of $M_{\text {dredge }}$ as a consequence of their thinner He-intershell regions. However, around 4-6 $M_{\odot}$ there is an increase in $M_{\text {dredge }}$ as a consequence of the models experiencing many more TPs relative to their lower mass counterparts. This means that the total yield of $\mathrm{C}+\mathrm{N}$, for example, will be higher in these models. Figure 1 will be useful later when discussing the behavior of the yields and final surface abundances as a function of mass and metallicity.

\subsection{Post-processing Nucleosynthesis Calculations}

Post-processing calculations have been performed on all of the canonical helium composition models from Karakas (2014) and all of the new models in Table 1.

The numerical method used for the post-processing numerical calculations is the same as described in previous studies including Lugaro et al. (2012), Fishlock et al. (2014), and Shingles et al. (2015). The reaction rates are from the JINA reaclib database, as for 2012 May, except for the neutron-capture cross section of the $\mathrm{Zr}$ isotopes, which were updated by Lugaro et al. (2014b). The one major difference here is that we have updated the nuclear network used in the calculations to include more nuclear species, 328 instead of the previous 320, and the description of the temperature dependence of $\beta$-decay rates for a number of unstable isotopes. This was done to follow in more detail the behavior of a selection of long lived radioactive isotopes and of branching points on the $s$-process path: specifically, ${ }^{107} \mathrm{Pd},{ }^{127,129} \mathrm{I}$, and 181,182 Hf from Lugaro et al. (2014a), as well as at 134,135,136,137 $\mathrm{Cs},{ }^{154,155} \mathrm{Eu}$, and ${ }^{160} \mathrm{~Tb}$.

Here we present elemental abundances for elements heavier than $\mathrm{Fe}$ and $\mathrm{Ni}$, for which the treatment of branching points does not have a major effect on the results. ${ }^{7}$ Results for isotopic ratios of elements heavier than iron can, however, be strongly affected by branching points. These are not presented here, and will be discussed instead in dedicated forthcoming papers

\footnotetext{
Except for the specific cases of $\mathrm{Rb}$ and and $\mathrm{Cs}$, which are affected by the branching points located at unstable nuclei on the $s$-process path at ${ }^{85} \mathrm{Kr},{ }^{86} \mathrm{Rb}$, and ${ }^{134} \mathrm{Cs}$ (all treated correctly in our network) and of $\mathrm{Tl}$, which can be mildly affected by the branching points at ${ }^{203} \mathrm{Hg}$ and ${ }^{204} \mathrm{Tl}$ (for which we have not yet implemented the predicted temperature dependence of the decay rates).
}

aimed at comparing our results with the isotopic compositions observed in meteoritic inclusions and stardust grains.

\subsection{The Inclusion of ${ }^{13} \mathrm{C}$ Pockets}

To match the observations that show that AGB stars, their progeny, and their companions are enriched in the abundances of the $s$-process elements by up to 1 dex at solar metallicity (see e.g., Busso et al. 2001; Abia et al. 2002), a large number of neutrons are needed to be released in the intershell via $(\alpha, \mathrm{n})$ reactions. The main source of neutrons is the ${ }^{13} \mathrm{C}(\alpha, \mathrm{n}){ }^{16} \mathrm{O}$ reaction, which is activated at relatively low temperatures of $\sim 90 \mathrm{MK}$. However, CN cycling does not leave enough ${ }^{13} \mathrm{C}$ nuclei in the He-intershell. The standard solution to this problem is to assume that some partial mixing occurs between the convective $\mathrm{H}$-rich envelope and the intershell at the deepest extent of each TDU, so that the protons are captured by ${ }^{12} \mathrm{C}$ to produce a region rich in ${ }^{13} \mathrm{C}$, the so-called ${ }^{13} \mathrm{C}$ "pocket." The inclusion of ${ }^{13} \mathrm{C}$ pockets in theoretical calculations of $\mathrm{AGB}$ stars is one of the most significant uncertainties affecting predictions of the $s$ process (see the discussion in Busso et al. 1999; Herwig 2005; Karakas \& Lattanzio 2014). Here, we adopt the same techniques we have applied before in, e.g., Fishlock et al. (2014).

Our method is to insert protons at the deepest extent of each TDU episode in the post-processing calculations. The protons are partially mixed over a mass extent in the intershell denoted by $M_{\text {mix }}$, using an exponentially declining profile such that at the base of the envelope the proton abundance is $X_{\mathrm{p}} \approx 0.7$ (i.e., the envelope hydrogen abundance) and in the intershell, at $M_{\text {mix }}$ below the base of the convective envelope, the hydrogen abundance is $X_{\mathrm{p}}=1 \times 10^{-4}$. Below this point in mass $X_{\mathrm{p}}=0$.

Our method differs from that of Cristallo et al. (2015), who include time-dependent convective overshoot in their models at the base of the envelope, which, at the deepest extent of each TDU episode, leads to the mixing of protons into the intershell that produces the ${ }^{13} \mathrm{C}$ pocket (Cristallo et al. 2009). This method is more self-consistent than ours, since we directly insert the proton abundance profile rather than the mixing process that leads to it. However, our $s$-process results for AGB stars of low mass, where ${ }^{13} \mathrm{C}$ is the main neutron source, are reasonably similar to those of Cristallo et al. (2015), as discussed by Lugaro et al. (2012), Fishlock et al. (2014), and in Section 5. This demonstrates that our parametric approach is a good reproduction of the self-consistent model of timedependent convective overshoot. While our method ignores any feedback from the partially $\mathrm{CN}$ cycling of the protons on the structure, it has the advantage that it allows us to easily adjust the $M_{\text {mix }}$ parameter and the proton profile and study the effect of their variations on AGB nucleosynthesis (e.g., Lugaro et al. 2014b, 2015).

Evidence for variations in the quantity and distribution of $s$ process elements come from a number of observational sources including post-AGB stars (e.g., Bonačić Marinović et al. 2007; De Smedt et al. 2012), AGB stars, and chemically peculiar stars that show the chemical signature of mass transfer from lowmass AGB stars (e.g., Ba and $\mathrm{CH}$ stars, carbon-enhanced, metal-poor stars; Busso et al. 2001; Bisterzo et al. 2011; Lugaro et al. 2012), as well as pre-solar grains (Lugaro et al. 2003, 2014b). These may be due to variation in the size of the ${ }^{13} \mathrm{C}$ pockets and/or in the profile of the proton abundance leading to their formation. However, they cannot be theoretically derived from first principles because we do not know the 
Table 2

Choice of $M_{\text {mix }}$ for Stellar Models in Different Mass Ranges

\begin{tabular}{lcccc}
\hline \hline$M_{\text {mix }} / M_{\odot}=$ & 0 & $1 \times 10^{-4}$ & $1 \times 10^{-3}$ & $2 \times 10^{-3}$ \\
\hline Standard & $M \geqslant 5 M_{\odot}$ & $4<M<5 M_{\odot}$ & $3<M \leqslant 4 M_{\odot}$ \\
\hline Extra models & $(4.25,0.03)$ & $(5,0.03)$ & $(3.0,0.007)$ & $(4.25,0.03)$ \\
& $(4.5,0.007)$ & $(4,0.007)$ & $(4.5,0.03)$ & $(3.25,0.03)$ \\
& $(4.75,0.007)$ & $\cdots$ & $\cdots$ & $\cdots$ \\
\hline
\end{tabular}

Note. We also show additional stellar models (mass in $M_{\odot}$, metallicity) calculated with different values of $M_{\text {mix }}$ for metallicities other than $Z=0.014$.

physical mechanism responsible for producing the partial mixing of protons into the top layers of the He-intershell. We refer to discussions in Herwig (2005) and Cristallo et al. (2009). Furthermore, stellar rotation has been also shown to affect the quantity and the distribution of the s-process elements produced in the ${ }^{13} \mathrm{C}$ pocket (Herwig et al. 2003; Siess et al. 2004; Piersanti et al. 2013). In this context, however, the effect of magnetic fields has not yet been investigated.

We include ${ }^{13} \mathrm{C}$ pockets in all the low-mass AGB models below $4.5 M_{\odot}$ that experience TDU. We do not include rotation or magnetic fields in our models and we keep the same exponential proton profile described above in all models and for all ${ }^{13} \mathrm{C}$ pockets; however, we experiment with changing the value of the $M_{\text {mix }}$ parameter to produce larger or smaller pockets, in terms of their extension in mass. The value of $M_{\text {mix }}$ was chosen as function of the stellar mass, with our standard choices for each mass range listed in Table 2. Additional models are calculated with different values of $M_{\text {mix }}$; in Table 3 we show the entire range of stellar nucleosynthesis models calculated for $Z=0.014$, which are the most extensive. In Table 2 we also provide a list of the additional models calculated for the other metallicities.

For stars of initial mass up to and equal to $3 M_{\odot}$, we used as the standard choice $M_{\text {mix }}=2 \times 10^{-3} M_{\odot}$, which results in a ${ }^{13} \mathrm{C}$ pocket mass typically about one-tenth of the mass of the He-rich intershell. This value of $M_{\text {mix }}$ is required to match the strong observational constraint that AGB stars of metallicity close to solar are enhanced in $s$-process elements by up to 1 dex, as demonstrated previously by, e.g., Gallino et al. (1998). Similar considerations were also used by Cristallo et al. (2009) to calibrate the value of the free parameter $\beta$ that controls the exponential decay of the velocity below the border of the convective envelope in their time-dependent description of overshoot.

For stars of initial mass between 3 and (including) $4 M_{\odot}$, we used as standard choice $M_{\text {mix }}=10^{-3} M_{\odot}$. This is because the mass of the intershell decreases and calculations including hydrodynamical overshoot indicate that $M_{\text {mix }}$ also follows such a decrease (Cristallo et al. 2009). For masses between 4.25 and $5 M_{\odot}$, we set $M_{\text {mix }}=10^{-4} M_{\odot}$ as our standard choice, an order of magnitude smaller than for the lower masses, again following the shrinking of the mass of the intershell. An exception is made for the $4.75 M_{\odot}, Z=0.007$ model, which we assume does not form ${ }^{13} \mathrm{C}$ pockets. This is motivated by the strong HBB experienced by this model, with temperatures reaching over $75 \mathrm{MK}$ at the base of the envelope.

For intermediate-mass AGB models above $5 M_{\odot}$, we do not include a ${ }^{13} \mathrm{C}$ pocket, following indications from theory (Goriely \& Siess 2004) and observations (García-Hernández et al. 2013) that the ${ }^{13} \mathrm{C}$ pocket is not present. Instead, in these models the $s$ process proceeds through activation of the ${ }^{22} \mathrm{Ne}$ $(\alpha, \mathrm{n})^{25} \mathrm{Mg}$ reaction inside the $\mathrm{TP}$, which requires temperatures
Table 3

The Stellar Nucleosynthesis Models Calculated for $Z=0.014$

\begin{tabular}{|c|c|c|c|c|c|}
\hline $\begin{array}{l}M_{\text {mix }} / M_{\odot}= \\
\text { Stellar } \\
\text { Mass }\left(M_{\odot}\right)\end{array}$ & 0 & $1 \times 1 \times 10^{-4}$ & $1 \times 10^{-3}$ & $2 \times 10^{-3}$ & $4 \times 10^{-3}$ \\
\hline 1.00 & $\checkmark$ & & & & \\
\hline 1.25 & $\checkmark$ & & & & \\
\hline 1.50 & $\sqrt{ }^{\mathrm{a}}$ & & & $\checkmark^{\mathrm{b}}[\mathrm{ST}]$ & \\
\hline 1.75 & $\sqrt{ }^{\mathrm{a}}$ & & & $\checkmark^{\mathrm{b}}[\mathrm{ST}]$ & \\
\hline 2.00 & & $\checkmark$ & $\checkmark$ & $\checkmark[\mathrm{ST}]$ & $\checkmark$ \\
\hline 2.25 & & & & $\checkmark[\mathrm{ST}]$ & \\
\hline 2.50 & & & & $\checkmark[\mathrm{ST}]$ & \\
\hline 2.75 & & & & $\checkmark[\mathrm{ST}]$ & \\
\hline 3.00 & & $\checkmark$ & $\checkmark$ & $\checkmark[\mathrm{ST}]$ & \\
\hline 3.25 & & & $\checkmark[\mathrm{ST}]$ & $\checkmark$ & \\
\hline 3.50 & & & $\checkmark[\mathrm{ST}]$ & & \\
\hline 3.75 & & & $\checkmark[\mathrm{ST}]$ & & \\
\hline 4.00 & & $\checkmark$ & $\checkmark[\mathrm{ST}]$ & & \\
\hline 4.25 & & $\checkmark[\mathrm{ST}]$ & $\checkmark$ & & \\
\hline 4.50 & $\checkmark$ & $\checkmark[\mathrm{ST}]$ & $\checkmark$ & & \\
\hline 4.75 & $\checkmark$ & $\checkmark[\mathrm{ST}]$ & & & \\
\hline 5.00 & $\checkmark[\mathrm{ST}]$ & $\checkmark$ & & & \\
\hline 5.50 & $\checkmark[\mathrm{ST}]$ & & & & \\
\hline 6.00 & $\checkmark[\mathrm{ST}]$ & & & & \\
\hline 7.00 & $\checkmark[\mathrm{ST}]$ & & & & \\
\hline 8.00 & $\checkmark[\mathrm{ST}]$ & & & & \\
\hline
\end{tabular}

Notes. A checkmark $(\checkmark)$ shows the the size of $M_{\text {mix }}$ used in the calculations. The [ST] label indicates the cases with the standard choice for each model with TDU.

${ }^{\mathrm{a}}$ For the model without overshoot.

${ }^{\mathrm{b}}$ For the model with overshoot.

in excess of 300 MK (Karakas et al. 2012; van Raai et al. 2012).

\section{SURFACE ABUNDANCES DURING THE AGB}

Here we present a summary of the results from the postprocessing nucleosynthesis calculations, starting with the surface abundances.

\subsection{The Surface Abundance Data Tables}

We provide three sets of tables, one for each metallicity: (1) the elemental surface abundances as a function of TP number for each $(M, Z)$ combination; (2) the isotopic ratios of the elements up to $\mathrm{Ni}$ as a function of TP number for each $(M, Z)$ combination; and (3) the integrated elemental yields. In this section we describe the contents of the first two tables (surface abundances and isotopic ratios), with the yield tables described in Section 4. 
For the $(M, Z)$ combinations in Table 1 where we include convective overshoot, we provide nucleosynthesis predictions from the case with and without overshoot. If there is no TDU in the model without convective overshoot (e.g., $1.5 M_{\odot}$, $Z=0.014)$, no ${ }^{13} \mathrm{C}$ pocket is included. For these $(M, Z)$ combinations, the value of $N_{\text {ov }}$ used in the calculation is provided in the header files. If no value of $N_{\mathrm{ov}}$ is specified, no overshoot is included.

We provide examples of each of the data file types. Table 4 illustrates the information included in the surface abundance data files. The surface abundance data tables start with the initial abundances used in the post-processing calculations and then include elemental abundances as a function of TP number. At each entry we include the TP number, the stellar mass, core mass, and envelope mass at that TP (in $M_{\odot}$ ), and the surface luminosity (in $\log L_{\odot}$ ). After the abundances of each element are given, we then provide the surface elemental ratios of $\mathrm{He} /$ $\mathrm{H}, \mathrm{C} / \mathrm{O}$, and $\mathrm{N} / \mathrm{O}$ at that TP. The final entry for each $(M, Z)$ combination is the final elemental abundances, computed at the last time step (which may fall on a TP or during the interpulse period).

For all elements except $\mathrm{Li}, \mathrm{B}$, and $\mathrm{B}$ we include the element name, the proton number, $z$, the abundance in the format $\log \epsilon(X)$ where $\log \epsilon(X)=\log _{10}(\mathrm{X} / \mathrm{H})+12 ;[\mathrm{X} / \mathrm{H}],{ }^{8}[\mathrm{X} /$ $\mathrm{Fe}$, [X/O], and the mass fraction $X(i)$. The radioactive elements Tc and Pm may have non-zero $\log \epsilon(X)$ values if they are produced in the He-intershell and dredged to the surface. Note that we do not decay the abundances of radioactive isotopes (e.g., ${ }^{26} \mathrm{Al},{ }^{60} \mathrm{Fe}$ ) in the isotopic or elemental surface abundance files, but they are assumed to have all decayed in the yield tables. However, we do decay the isotope ${ }^{93} \mathrm{Zr}$ to ${ }^{93} \mathrm{Nb}$ because $\mathrm{Nb}$ obtains essentially all of its production via this decay.

We do not include the surface abundances and yields for Be and $\mathrm{B}$ because these elements are not synthesized in stars. Theoretical predictions for $\mathrm{Li}$ are highly dependent on the numerical procedure, as demonstrated by Lattanzio et al. (2015) for low-mass RGB stars with thermohaline mixing. The Li abundances in low-mass stars is altered by thermohaline mixing or some other deep mixing process on the RGB, which we do not include here. This means our models of low-mass AGB stars begin the AGB with incorrect $\mathrm{Li}$ abundances, implying that our AGB yields will similarly be incorrect. Note that while this problem also affects the ${ }^{12} \mathrm{C} /{ }^{13} \mathrm{C}$ ratio, it is possible to correct for the effects of extra mixing on the evolution of the ${ }^{12} \mathrm{C} /{ }^{13} \mathrm{C}$ ratio (e.g., Lebzelter et al. 2008; Karakas et al. 2010). This is not possible for Li because of the uncertainties affecting numerical predictions. Li abundance predictions from models with HBB have been shown to be consistent with observations of bright O-rich stars in the Magellanic Clouds and Galaxy (e.g., Lattanzio et al. 1997; Ventura et al. 2000; van Raai et al. 2012; García-Hernández et al. 2013). This suggests that predictions are more robust in intermediate-mass models, where production can be copious. For this reason we provide lithium abundances and yields from models with $\mathrm{HBB}$ in Table 5, with the results discussed in Section 3.2.

\footnotetext{
8 Where we use the standard spectroscopic notation, [A/ $\mathrm{B}]=\log _{10}(A / B)_{\text {surf }}-\log _{10}(A / B)_{\odot}$. The ratio $(A / B)_{\text {surf }}$ is the number ratio of elements $A$ and $B$ at the surface of the model star and $(A / B)_{\odot}$ is the solar number ratio, taken from Asplund et al. (2009).
}

In the isotopic data tables we provide the following isotopic ratios, many of which are useful for comparison to stellar/PNs spectra or stardust grains: ${ }^{12} \mathrm{C} /{ }^{13} \mathrm{C},{ }^{14} \mathrm{~N} /{ }^{15} \mathrm{~N},{ }^{16} \mathrm{O} /{ }^{17,18} \mathrm{O},{ }^{24} \mathrm{Mg} /$ ${ }^{25,26} \mathrm{Mg},{ }^{26} \mathrm{Al} /{ }^{27} \mathrm{Al},{ }^{28} \mathrm{Si} /{ }^{29,30} \mathrm{Si},{ }^{36,37} \mathrm{Cl} /{ }^{35} \mathrm{Cl},{ }^{36,38} \mathrm{Ar} /{ }^{40} \mathrm{Ar}$, ${ }^{40,41} \mathrm{~K} /{ }^{39} \mathrm{~K}, \quad{ }^{42,43,44,46,48} \mathrm{Ca} /{ }^{40} \mathrm{Ca}, \quad{ }^{46,47,49,50} \mathrm{Ti} /{ }^{48} \mathrm{Ti}, \quad{ }^{53,54} \mathrm{Cr} /$ ${ }^{52} \mathrm{Cr},{ }^{54,57,58,60} \mathrm{Fe} /{ }^{56} \mathrm{Fe}$, and ${ }^{60,61,62} \mathrm{Ni} /{ }^{58} \mathrm{Ni}$. In Table 6 we show an example of the isotopic data files available for each model, this time for the $3.5 M_{\odot}, Z=0.03$ model. We include only five isotopic ratios for illustrative purposes over the first three TPs. The tables finish with the final isotopic ratios calculated at the last time step.

\subsection{Elements from Helium to Iron}

In Table 5 we present $\mathrm{Li}$ abundances and stellar yields for models that experience HBB. We include the peak surface abundance of lithium, the TP number when the peak abundance occurs, the mass of lithium expelled from the star (in $M_{\odot}$ ), and the initial mass of lithium present in the wind (in $M_{\odot}$ ). All of the models in Table 5 experience HBB, as noted in Karakas (2014), with the exception of the $4.75 M_{\odot}, Z=0.03$ model. In Karakas (2014), the minimum temperature at the base of the envelope for $\mathrm{HBB}$ was arbitrarily set at $50 \mathrm{MK}$, which seems to be the minimum required to see the effects of $\mathrm{CN}$ cycling at the surface. The peak temperature in the $4.75 M_{\odot}, Z=0.03$ model is $42.5 \mathrm{MK}$, which is hot enough to produce some $\mathrm{Li}$ with a peak $\log \epsilon(\mathrm{Li})=3.0$. Table 5 shows that the peak $\mathrm{Li}$ abundance depends on the initial metallicity, with the most massive metal-rich $Z=0.03$ models predicting super-Li-rich AGB stars with $\log \epsilon(\mathrm{Li}) \geqslant 5$. The table also shows that the peak abundance occurs earlier in the evolution when the stellar mass increases, with models $M \geqslant 7 M_{\odot}$ experiencing the highest abundance at the first TP.

In Figures 2-4 we show the $\mathrm{He} / \mathrm{H}, \mathrm{C} / \mathrm{O}$, and $\mathrm{N} / \mathrm{O}$ ratios from the surface of the $Z=0.014, Z=0.03$, and $Z=0.007$ models. We include models with convective overshoot where applicable. We plot the ratios after the first TP and after the last TP. The ratio after the first TP reflects surface abundance changes prior to the TP-AGB. This includes the first dredge up (FDU), which occurs in all models, although the surface abundance changes are strongest around 2-3 $M_{\odot}$ (Boothroyd \& Sackmann 1999) and the SDU. SDU occurs in models more massive than about $4-5 M_{\odot}$, depending on $Z$. We refer to Table 1 and Table 1 from Karakas (2014) for the minimum masses for SDU and HBB. The 7 and $8 M_{\odot}$ models begin HBB before the first $\mathrm{TP}$, and this is reflected in the lower $\mathrm{C} / \mathrm{O}$ and higher $\mathrm{N} / \mathrm{O}$ ratios.

In Figures $5-7$ we show the ratios of ${ }^{12} \mathrm{C} /{ }^{13} \mathrm{C},{ }^{14} \mathrm{~N} /{ }^{15} \mathrm{~N}$, ${ }^{17} \mathrm{O} /{ }^{18} \mathrm{O}$, and ${ }^{25} \mathrm{Mg} /{ }^{26} \mathrm{Mg}$ at the first TP and after the last TP for the $Z=0.014, Z=0.03$, and $Z=0.007$ models. We do not include any thermohaline mixing or other form of nonconvective extra mixing into our calculations of RGB and AGB envelopes. This mean that our, e.g., ${ }^{12} \mathrm{C} /{ }^{13} \mathrm{C}$ ratios in low-mass $\left(M \lesssim 2 M_{\odot}\right)$ models are higher than measured in RGB stars (see the discussions in Charbonnel 1994; Charbonnel \& Zahn 2007; Eggleton et al. 2008; Karakas \& Lattanzio 2014). It is unclear how much extra mixing occurs in the envelopes of solar-metallicity AGB stars that become carbon-rich. Extra mixing on the RGB and TDU on the AGB can account for the majority of the observed ${ }^{12} \mathrm{C} /{ }^{13} \mathrm{C}$ ratios on the AGB (Karakas et al. 2010). There are exceptions including a small sample of C-rich stars with low ${ }^{12} \mathrm{C} /{ }^{13} \mathrm{C}$ ratios $<30$ and the J-star population (Abia \& Isern 1997; Lebzelter et al. 2008). 
Table 4

Example of the Surface Abundance Tables Available

\begin{tabular}{|c|c|c|c|c|c|c|}
\hline \\
\hline \multicolumn{7}{|c|}{$\begin{array}{l}\# \\
\# \text { Initial mass }=3.500, Z=0.0300, Y=0.300, M_{\text {mix }}=1.00 \mathrm{E}-03 \\
\#\end{array}$} \\
\hline \multicolumn{7}{|c|}{$\begin{array}{l}\text { \# TP } \text { Mass } \text { Mcore Menv } \log L \\
\text { \# } 153.4980700 .6940672 .8040004 .096260\end{array}$} \\
\hline \#El & $Z$ & $\log e(X)$ & {$[\mathrm{X} / \mathrm{H}]$} & [X/Fe] & [X/O] & $X(i)$ \\
\hline c & 6 & 8.799475 & 0.218580 & -0.121316 & -0.101519 & $3.83509 \mathrm{E}-03$ \\
\hline $\mathrm{n}$ & 7 & 8.199430 & 0.774327 & 0.434431 & 0.454228 & 4.03913E-03 \\
\hline o & 8 & 9.059475 & 0.320489 & -0.019408 & 0.000000 & $1.17543 \mathrm{E}-02$ \\
\hline $\mathrm{f}$ & 9 & 4.789476 & 0.370921 & 0.031024 & 0.050432 & $8.41870 \mathrm{E}-07$ \\
\hline
\end{tabular}

Note. We show the first few lines at the beginning of the $3.5 M_{\odot}, Z=0.03$ model table, and the first few lines after thermal pulse \#15.

(This table is available in its entirety in FITS format.)

Table 5

Peak Surface Lithium Abundance During the AGB and Stellar Yields from Models with Hot Bottom Burning

\begin{tabular}{|c|c|c|c|c|c|}
\hline $\begin{array}{l}\text { Initial Mass } \\
\left(M_{\odot}\right)\end{array}$ & $Z$ & $\begin{array}{l}\text { Peak Lithium Abundance } \\
\qquad \log \epsilon(\mathrm{Li})\end{array}$ & TP at Peak & $\begin{array}{l}\text { Mass Li Expelled } \\
\qquad\left(M_{\odot}\right)\end{array}$ & $\begin{array}{c}\text { Initial } \mathrm{Li} \text { in Wind } \\
\qquad\left(M_{\odot}\right)\end{array}$ \\
\hline 4.75 & 0.03 & 3.00 & 15 & $1.7599538 \mathrm{E}-07$ & $6.5591479 \mathrm{E}-08$ \\
\hline 5.00 & 0.03 & 4.03 & 15 & $2.3315576 \mathrm{E}-07$ & $6.9651541 \mathrm{E}-08$ \\
\hline 5.50 & 0.03 & 4.70 & 14 & $1.4348146 \mathrm{E}-07$ & $7.7758202 \mathrm{E}-08$ \\
\hline 6.00 & 0.03 & 4.87 & 12 & $1.8557853 \mathrm{E}-07$ & $8.5831154 \mathrm{E}-08$ \\
\hline 7.00 & 0.03 & 5.18 & 5 & 2.3292797E-07 & $1.0173942 \mathrm{E}-07$ \\
\hline 8.00 & 0.03 & 5.18 & 1 & $5.1344944 \mathrm{E}-07$ & $1.1707634 \mathrm{E}-07$ \\
\hline 4.25 & 0.014 & 3.80 & 19 & 4.1797335E-08 & $3.0904165 \mathrm{E}-08$ \\
\hline 4.50 & 0.014 & 3.90 & 19 & $3.2354016 \mathrm{E}-08$ & $3.3096789 \mathrm{E}-08$ \\
\hline 4.75 & 0.014 & 3.95 & 18 & $2.8601756 \mathrm{E}-08$ & 3.5298505E-08 \\
\hline 5.00 & 0.014 & 4.03 & 15 & $1.0902397 \mathrm{E}-08$ & 3.7490221E-08 \\
\hline 5.50 & 0.014 & 4.17 & 13 & 1.7997854E-09 & $4.1848171 \mathrm{E}-08$ \\
\hline 6.00 & 0.014 & 4.28 & 10 & 7.7072976E-10 & 4.6206125E-08 \\
\hline 7.00 & 0.014 & 4.40 & 5 & $9.1141705 \mathrm{E}-10$ & 5.4802843E-08 \\
\hline 8.00 & 0.014 & 4.43 & 1 & $3.0000244 \mathrm{E}-08$ & 6.3114797E-08 \\
\hline 4.00 & 0.007 & 2.91 & 18 & $9.1520405 \mathrm{E}-08$ & $1.4334581 \mathrm{E}-08$ \\
\hline 4.25 & 0.007 & 3.36 & 16 & 4.0745888E-08 & $1.5426464 \mathrm{E}-08$ \\
\hline 4.50 & 0.007 & 4.01 & 15 & $8.2011226 \mathrm{E}-10$ & $1.6500147 \mathrm{E}-08$ \\
\hline 4.75 & 0.007 & 4.03 & 14 & $3.9599790 \mathrm{E}-10$ & $1.7592029 \mathrm{E}-08$ \\
\hline 5.00 & 0.007 & 4.06 & 13 & $1.9964203 \mathrm{E}-10$ & $1.8674355 \mathrm{E}-08$ \\
\hline 5.50 & 0.007 & 3.62 & 12 & $2.1940057 \mathrm{E}-11$ & 2.0830822E-08 \\
\hline 6.00 & 0.007 & 4.42 & 7 & $4.0835935 \mathrm{E}-11$ & $2.2946342 \mathrm{E}-08$ \\
\hline 7.00 & 0.007 & 4.46 & 1 & $5.8903787 \mathrm{E}-10$ & 2.7113233E-08 \\
\hline 7.50 & 0.007 & 4.20 & 1 & $2.2296360 \mathrm{E}-08$ & 2.9042221E-08 \\
\hline
\end{tabular}

For these objects, some form of extra mixing on the AGB is required although the mechanism responsible is not known (and it is probably not thermohaline mixing; see Busso et al. 2010; Stancliffe 2010). Extra mixing also on the AGB has been invoked to explain the composition of roughly $10 \%$ of meteoritic stardust oxide grains (the Group 2 grains), which show depletions in ${ }^{18} \mathrm{O}$ (Nollett et al. 2003; Palmerini et al. 2011).

Halabi \& Eid (2015) perform a comprehensive analysis of model predictions against observations of $\mathrm{C}, \mathrm{N}$, and $\mathrm{O}$ isotopic ratios of Galactic RGB stars. In their study, they found agreement between their solar-like composition models spanning a range from 1.2 to $7 M_{\odot}$ and the predictions in Karakas \& Lattanzio (2014) for the ${ }^{16} \mathrm{O} /{ }^{17} \mathrm{O}$ ratio after first and second dredge. The predictions illustrated in Figure 5 are specifically for the AGB phase but the abundances at the first TP are consistent with the post-FDU and SDU abundances from the $Z=0.02$ models from Karakas \& Lattanzio (2014). The exceptions are for the $7 M_{\odot}$ and $8 M_{\odot}$ models because both of these cases start to show the effects of HBB between the 
Table 6

Example of the Isotopic Abundance Tables Available

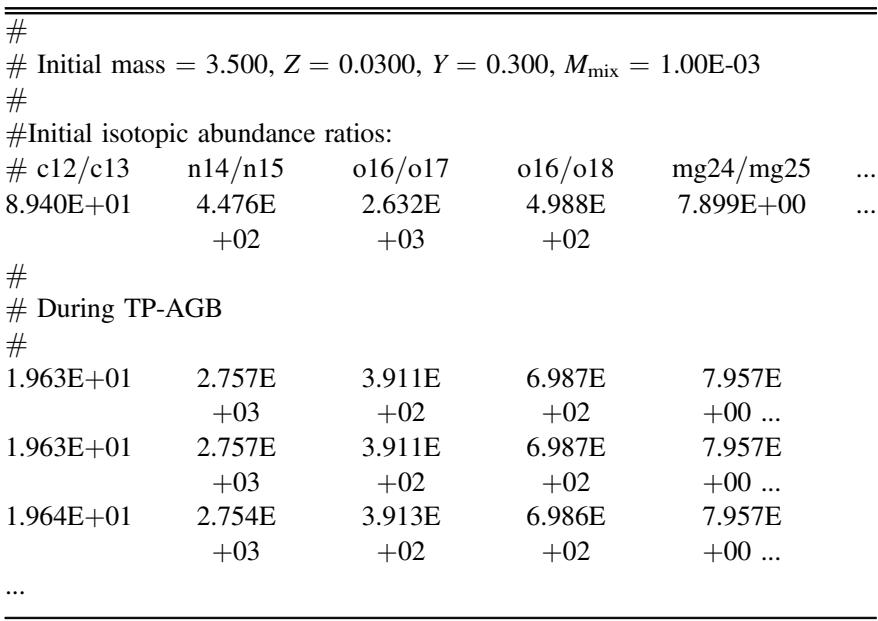

Note. We show the first few lines of the $3.5 M_{\odot}, Z=0.03$ model table for the first five isotopic ratios in the table.

(This table is available in its entirety in FITS format.)
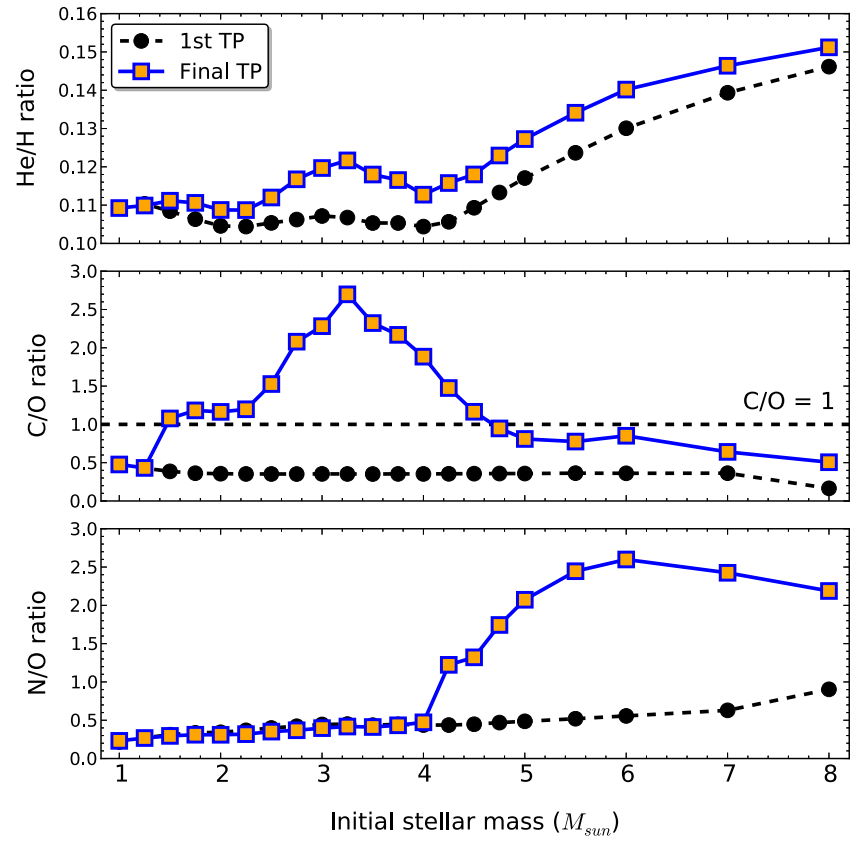

Figure 2. Ratios of $\mathrm{He} / \mathrm{H}, \mathrm{C} / \mathrm{O}$, and $\mathrm{N} / \mathrm{O}$ at the surface after the first thermal pulse and after the last thermal pulse for the $Z=0.014$ models.

deepest extent of SDU and the first TP. The ratios affected include ${ }^{12} \mathrm{C} /{ }^{13} \mathrm{C},{ }^{16} \mathrm{O} /{ }^{18} \mathrm{O}$, and ${ }^{14} \mathrm{~N} /{ }^{15} \mathrm{~N}$. For example, at the deepest extent of SDU, the ${ }^{12} \mathrm{C} /{ }^{13} \mathrm{C}=19.3$ at the surface of the $8 M_{\odot}, Z=0.014$ model; this drops to 2.64 by the start of the thermally pulsing phase.

As discussed by Halabi \& Eid (2015), there are few observational constraints on the ${ }^{14} \mathrm{~N} /{ }^{15} \mathrm{~N}$ ratio, especially for RGB stars. Hedrosa et al. (2013) measured the ${ }^{14} \mathrm{~N} /{ }^{15} \mathrm{~N}$ ratio in a sample of AGB stars and found evidence that some ${ }^{15} \mathrm{~N}$ production occurs in C-rich AGB stars. Figures 5-7 show that the ${ }^{14} \mathrm{~N} /{ }^{15} \mathrm{~N}$ ratio decreases between the first TP and the tip of the AGB for models that become C-rich. This indicates that some ${ }^{15} \mathrm{~N}$ production is happening in our models, albeit at a lower level than needed by the observations. The minimum value reached in our models is 1400 , while some stars have
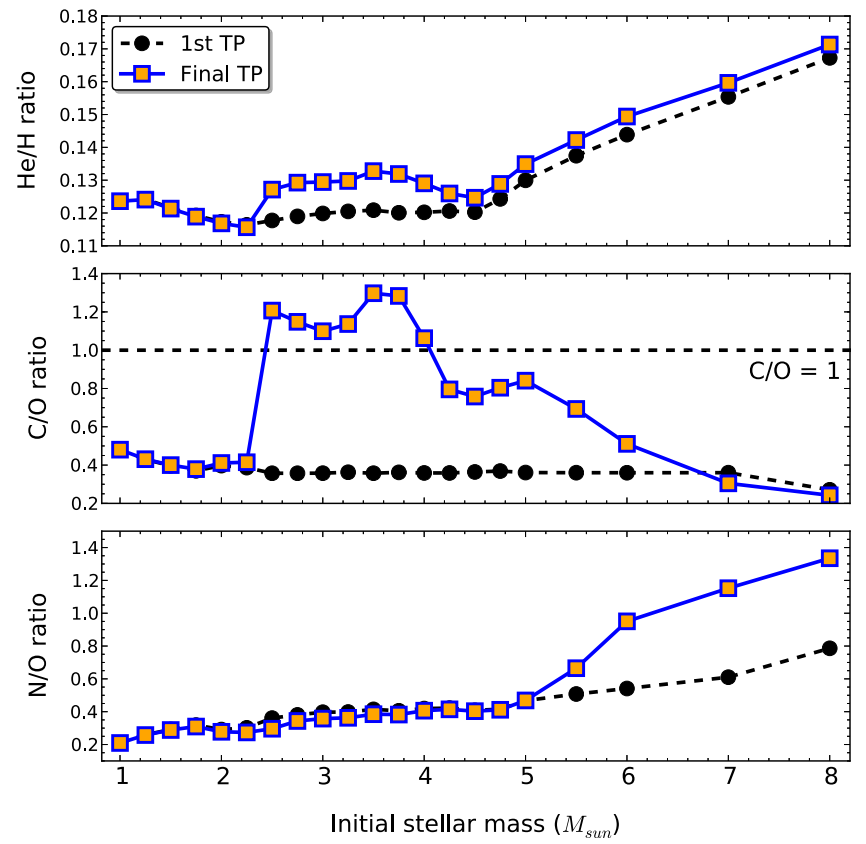

Figure 3. Same as Figure 2 except for the $Z=0.03$ models.
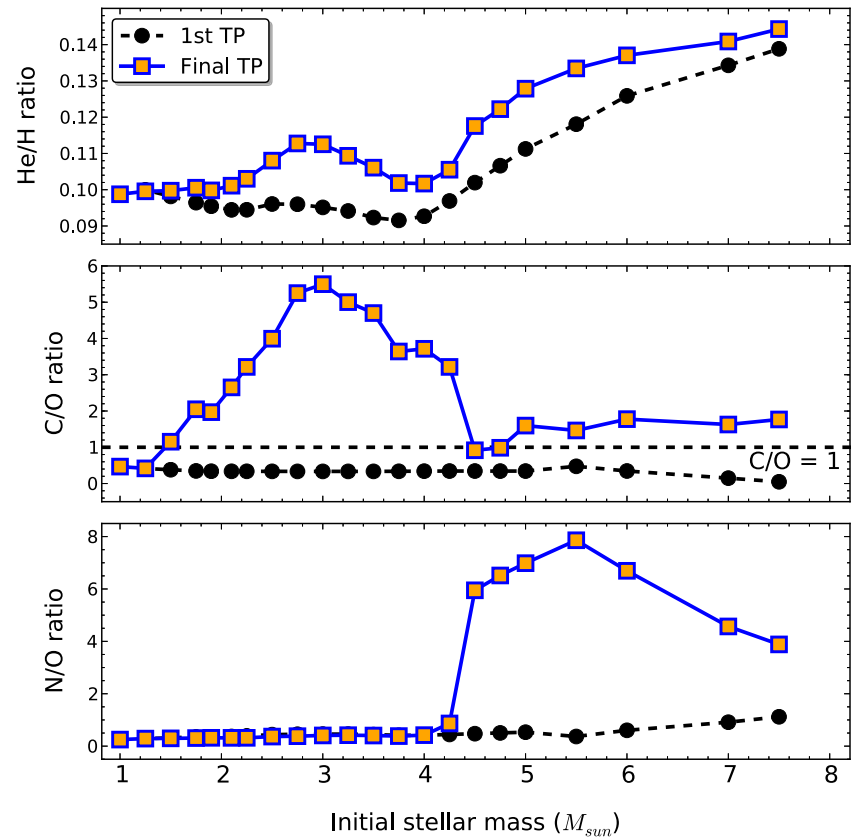

Figure 4. Same as Figure 2 except for the $Z=0.007$ models.

ratios $<1000$. The complex reaction pathway that produces ${ }^{19} \mathrm{~F}$ (Lugaro et al. 2004) first produces ${ }^{15} \mathrm{~N}$ as an intermediate step. In models where the nitrogen isotopic ratio decreases during the AGB, some of the ${ }^{15} \mathrm{~N}$ survives He-shell burning. In higher mass models, the ${ }^{15} \mathrm{~N}$ is destroyed to make ${ }^{19} \mathrm{~F}$ (which itself may be destroyed by $\alpha$ capture), or HBB destroys ${ }^{15} \mathrm{~N}$ by proton-capture in the envelope. Uncertainties in the reaction rates involved in this path may play a role, specifically the ${ }^{15} \mathrm{~N}(\alpha, \gamma){ }^{19} \mathrm{~F}$ reaction.

The oxygen isotope ratios have been measured in RGB and AGB stars. The most comprehensive study was by Harris and collaborators using high-resolution near-IR spectra (Harris \& Lambert 1984; Harris et al. 1985a, 1985b, 1987) for a sample 

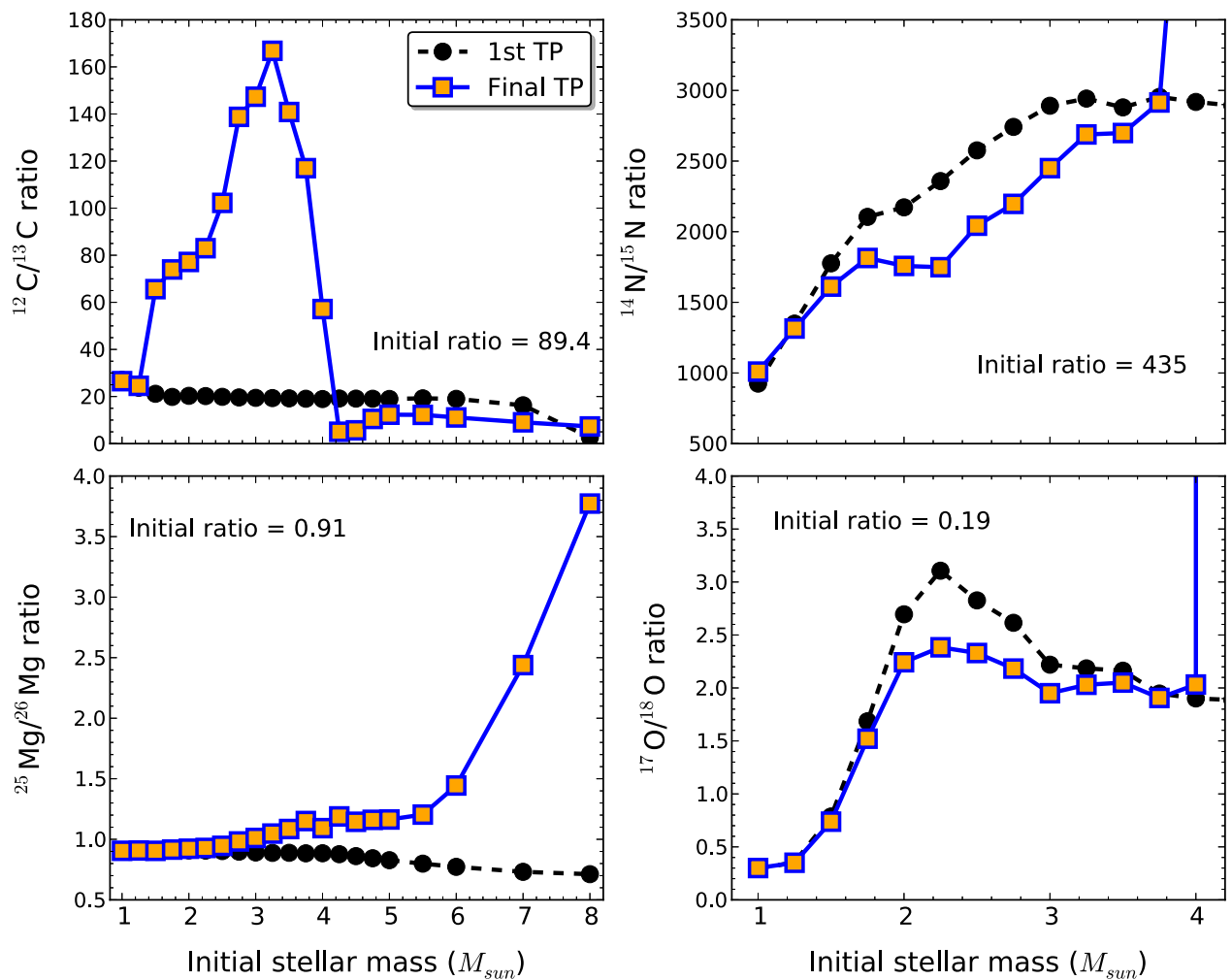

Figure 5. Ratios of ${ }^{12} \mathrm{C} /{ }^{13} \mathrm{C},{ }^{14} \mathrm{~N} /{ }^{15} \mathrm{~N},{ }^{17} \mathrm{O} /{ }^{18} \mathrm{O}$, and ${ }^{25} \mathrm{Mg} /{ }^{26} \mathrm{Mg}$ at the first thermal pulse and after the last thermal pulse for the $Z=0.014$ models. For the ${ }^{14} \mathrm{~N} /{ }^{15} \mathrm{~N}$ and ${ }^{17} \mathrm{O} /{ }^{18} \mathrm{O}$ ratios, we show results for models without $\mathrm{HBB}$, which includes masses up to $4 M_{\odot}$. The initial ratio is indicated on each panel.

of G, K, and M giants. Lebzelter et al. (2015) used near-IR spectra to measure oxygen in a small sample of RGB cluster stars covering a range of mass from $1.8-4.5 M_{\odot}$. They found good agreement with the theoretical models of Cristallo et al. (2011) and the observed ${ }^{16} \mathrm{O} /{ }^{17} \mathrm{O}$ ratio, but not with ${ }^{16} \mathrm{O} /{ }^{18} \mathrm{O}$. Many studies have focused on measuring the ${ }^{17} \mathrm{O} /{ }^{18} \mathrm{O}$ ratio from CO observations in mass-losing AGB stars (Kahane et al. 1992, 2000; Decin et al. 2010; Khouri et al. 2014), which is why we show show this ratio in Figures 5-7. Kahane et al. (1992) find values between 1.12 and 1.66 for the ${ }^{17} \mathrm{O} /{ }^{18} \mathrm{O}$ ratio, which suggests stars with masses between $\approx 1.5 M_{\odot}$ to $2 M_{\odot}$ at solar metallicity (or between 2 and $3 M_{\odot}$ if the stars have a metallicity that is $0.03 \approx 2 \mathrm{Z}_{\odot}$ ). The ${ }^{17} \mathrm{O} /{ }^{18} \mathrm{O}$ ratios estimated from the data of Lebzelter et al. (2015) range from 1.08 for Star 415 in NGC 7789, with an estimated mass of $1.8 M_{\odot}$, to 1.42 for HD 16068 in $\operatorname{Tr} 2$, which has an estimated mass of $4.5 M_{\odot}$. The observed ratios are lower than the predictions in Figures 5 for their estimated mass, indicating that higher initial ${ }^{18} \mathrm{O}$ abundance may be required, as also discussed by Lebzelter et al. (2015).

Similar to the case for the nitrogen isotopic ratio, the model predictions illustrated in Figures 5-7 show that the ${ }^{17} \mathrm{O} /{ }^{18} \mathrm{O}$ ratio decreases from the first TP to the tip of the AGB for models that become C-rich and do not experience HBB. The decrease occurs because ${ }^{18} \mathrm{O}$ is not completely destroyed by ${ }^{18} \mathrm{O}(\alpha, \gamma){ }^{22} \mathrm{Ne}$ during TPs and is therefore dredged to the stellar surface by TDU. For models with HBB, efficient destruction of ${ }^{18} \mathrm{O}$ leads to very high predicted ratios. Measurements of the oxygen isotope ratio in bright O-rich AGB stars have been attempted (Justtanont et al. 2015), with the result of a nondetection of ${ }^{18} \mathrm{O}$ as evidence for the existence of $\mathrm{HBB}$, which is consistent with calculations (see also Justtanont et al. 2013).
Solar metallicity models near the minimum mass for HBB $\left(M \lesssim 4.75 M_{\odot}\right)$ show final surface abundances that are consistent with J-type AGB stars and also a fraction of stardust silicon carbide ( $\mathrm{SiC}$ ) grains (the $\mathrm{A}+\mathrm{B}$ grains), where $\mathrm{C} / \mathrm{O} \gtrsim 1$ and ${ }^{12} \mathrm{C} /{ }^{13} \mathrm{C} \leqslant 10$ (Amari et al. 2001b). These conditions are not met in models of higher metallicity (e.g., see Figures 3 and 6 for the $Z=0.03$ models). The final surface composition of the intermediate-mass $Z=0.007$ models also have $\mathrm{C} / \mathrm{O}>1$ and low ${ }^{12} \mathrm{C} /{ }^{13} \mathrm{C}$ ratios, but the measured silicon isotopic ratios of $\mathrm{A}+\mathrm{B}$ grains suggest that they are mostly made in solarmetallicity stars (Amari et al. 2001b). Note, however, that models with $\mathrm{HBB}$ and $\mathrm{C} / \mathrm{O} \gtrsim 1$ also show high nitrogen and oxygen isotopic ratios. The nitrogen isotopic ratio in $\mathrm{A}+\mathrm{B}$ grains covers orders of magnitudes, from $\sim 30$ to $\sim 12,000$, and the grains with the highest ratios may be compatible with these intermediate-mass models. This ratio is difficult to measure in real stars and would be especially challenging in bright AGB stars with HBB. We predict that ${ }^{15} \mathrm{~N}$ would not be detected, making a determination of the nitrogen isotopic ratio impossible.

The final ${ }^{25} \mathrm{Mg} /{ }^{26} \mathrm{Mg}$ ratio is greater than solar in all the models and strongly increases with the initial mass as illustrated in, e.g., Figure 5 for the solar metallicity models. In the models without $\mathrm{HBB}$, it is dominated by the production of these isotopes in the intershell via ${ }^{22} \mathrm{Ne}+\alpha$ reactions (Karakas et al. 2006). In models with $\mathrm{HBB}$, the ${ }^{25} \mathrm{Mg} /{ }^{26} \mathrm{Mg}$ ratio is also affected by proton captures and the operation of the $\mathrm{MgAl}$ chain. In both cases, production of ${ }^{25} \mathrm{Mg}$ is favored consistent with the models of Ventura et al. (2013).

Depending on the initial stellar mass, several isotopic ratios of the elements from $\mathrm{Al}$ to $\mathrm{Ni}$ are predicted to show large variations. In Figure 8 we present four examples of these 

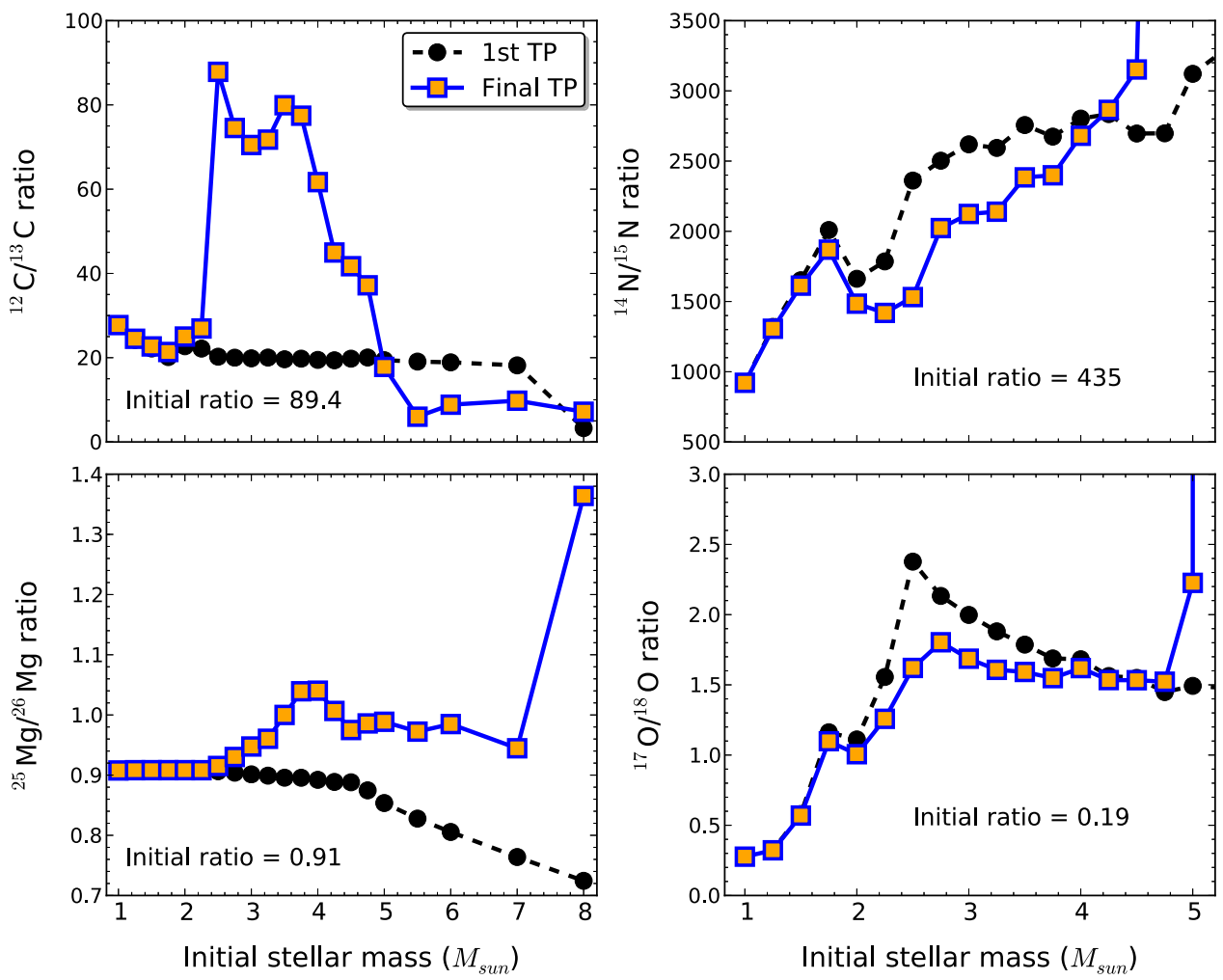

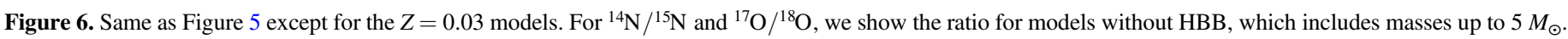
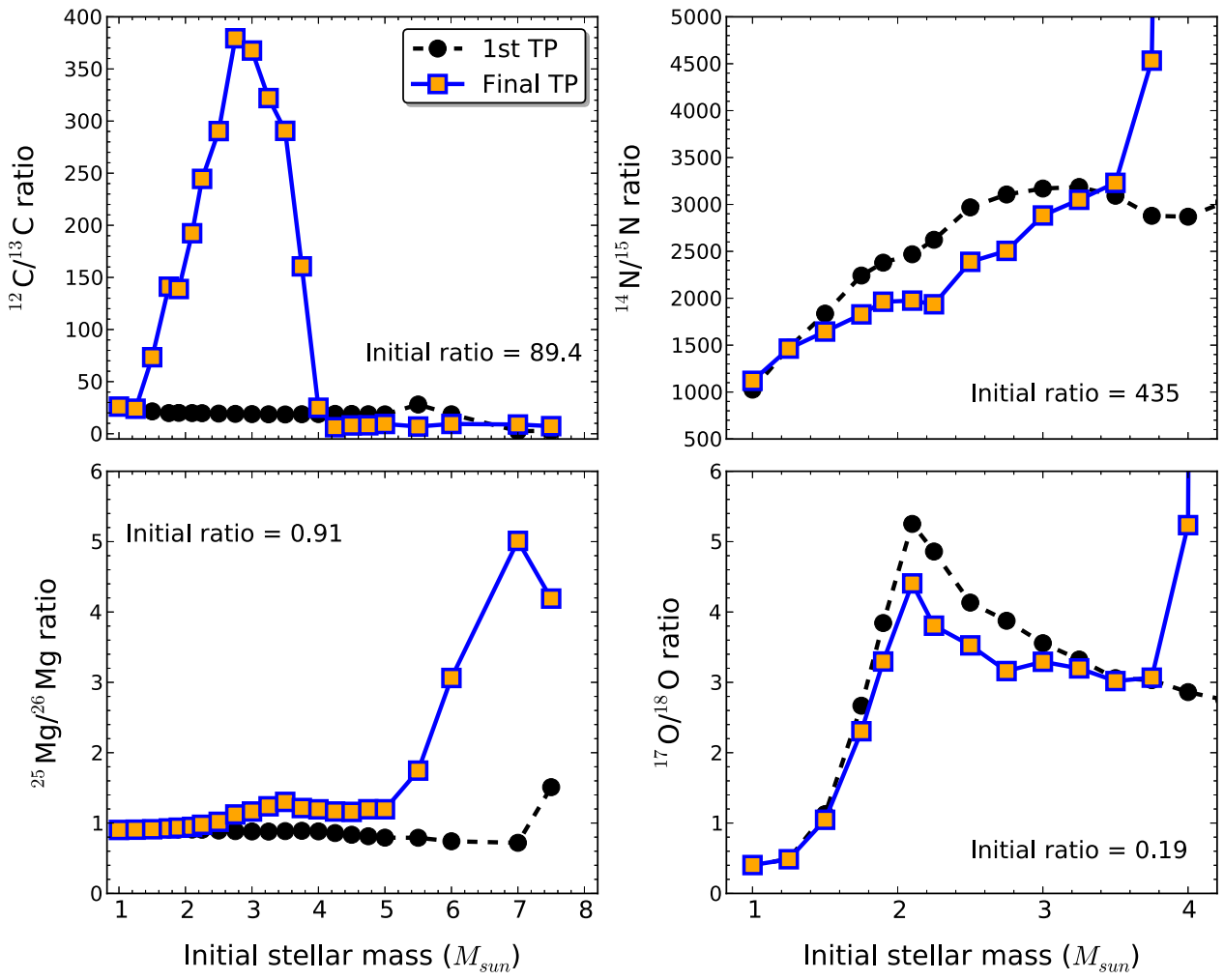

Figure 7. Same as Figure 5 except for the $Z=0.007$ models.

isotopic ratios which are modified by AGB nucleosynthesis. These can be measured in meteoritic stardust grains (e.g., Amari et al. 2001a), Furthermore, the Si isotopic ratios can be measured in AGB stars via molecular lines (e.g.,from $\mathrm{SiO}$,
Peng et al. 2013). The typical value of the ${ }^{26} \mathrm{Al} /{ }^{26} \mathrm{Al}$ ratio in AGB stars that do not experience HBB is between $10^{-3}$ and $10^{-2}$. This generally matches the range covered by oxide grains from O-rich AGB stars and by $\mathrm{SiC}$ grains from $\mathrm{C}$-rich $\mathrm{AGB}$ 

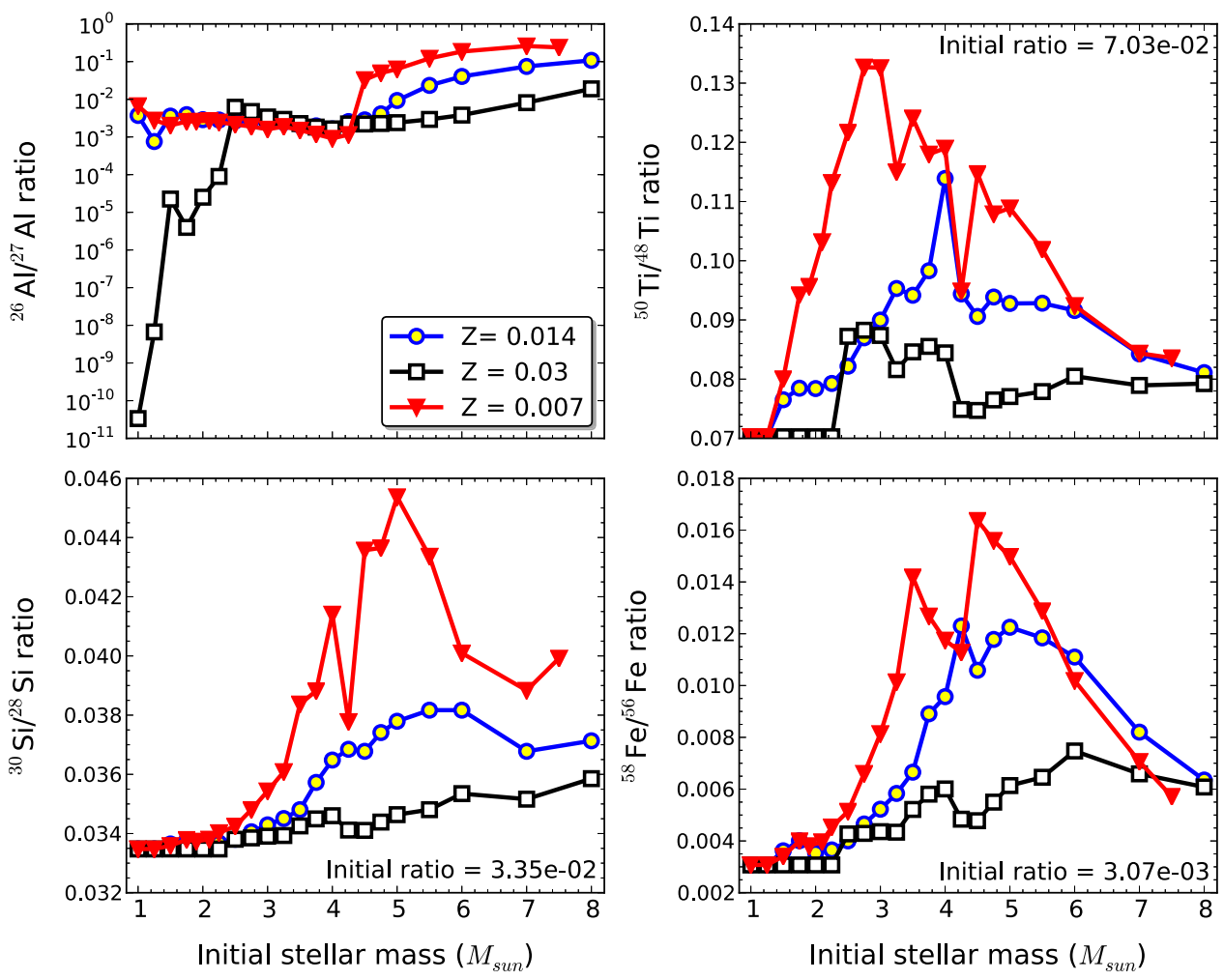

Figure 8. Four selected examples of surface isotopic ratios after the final TP as function of the initial stellar mass for all three metallicities.

stars, although it is not clear how to obtain values as low as $10^{-4}$, which have been detected in some grains (van Raai et al. 2008).

Most of the ${ }^{26} \mathrm{Al}$ at the stellar surface in these low-mass AGB models is the result of the TDU, where ${ }^{26} \mathrm{Al}$ in the intershell comes from the ingestion of the H-burning ashes. In the TP, ${ }^{26} \mathrm{Al}$ can be destroyed by neutron captures if the ${ }^{22} \mathrm{Ne}$ neutron source is activated because the ${ }^{26} \mathrm{Al}(\mathrm{n}, \mathrm{p})^{26} \mathrm{Mg}$ and ${ }^{26} \mathrm{Al}(\mathrm{n}, \alpha){ }^{23} \mathrm{Na}$ reactions have relatively high cross-sections ( $\sim 200$ mbarn). The slight decrease of ${ }^{26} \mathrm{Al} /{ }^{27} \mathrm{Al}$ with stellar mass and the difference between the $Z=0.03$ models and the other metallicities is consistent with the fact that the temperature at the base of the TP and the activation of the ${ }^{22} \mathrm{Ne}$ source increases with mass and decreases with metallicity. For models with $Z=0.03$ and mass below $2.5 M_{\odot}$, the ${ }^{26} \mathrm{Al} /{ }^{27} \mathrm{Al}$ ratio is much lower because they do not experience efficient TDU. Because the initial abundance of ${ }^{26} \mathrm{Al}$ is zero, it is extremely sensitive to the exact depth of the penetration of the convective envelope during the TDU, even just into the tip of the thin region of H-burning ashes that is not mixed into the TP. This explains why the 1 and $1.25 M_{\odot}$ models of $Z=0.007$ and $Z=0.014$ show higher ${ }^{26} \mathrm{Al} /{ }^{27} \mathrm{Al}$ ratios than the models of the same mass but $Z=0.03$, and why the $Z=0.03$ models of mass below $2.5 M_{\odot}$ show some variations with increasing the stellar mass. Above roughly $4 M_{\odot}, \mathrm{HBB}$ is the dominant production channel for ${ }^{26} \mathrm{Al}$, and the ${ }^{26} \mathrm{Al} /{ }^{27} \mathrm{Al}$ ratio reaches above 0.1 . The ${ }^{26} \mathrm{Al} /{ }^{27} \mathrm{Al}$ ratio grows with the temperature at the base of the envelope, which increases with increasing stellar mass and decreasing metallicity.

In contrast to ${ }^{26} \mathrm{Al} /{ }^{27} \mathrm{Al}$, the other three isotopic ratios shown in Figure 8 are only affected by neutron captures. This is generally the case for the isotopic ratios of the elements below $\mathrm{Fe}$ in AGB stars, particularly at the metallicities discussed here.
The ${ }^{30} \mathrm{Si} /{ }^{28} \mathrm{Si}$ and the ${ }^{58} \mathrm{Fe} /{ }^{56} \mathrm{Fe}$ ratios are mainly affected by the neutrons released in the TPs by the ${ }^{22} \mathrm{Ne}$ neutron source, and this is also generally the case for most of the isotopic ratios of the elements below Fe in AGB stars. This results in the largest changes observed at higher masses, with a peak around 5-6 $M_{\odot}$, depending on the metallicity. The maximum variations for the ${ }^{30} \mathrm{Si} /{ }^{28} \mathrm{Si}$ ratio is only $40 \%$, while for the ${ }^{58} \mathrm{Fe} /{ }^{56} \mathrm{Fe}$ ratio it is a factor of 5 . This is because the neutroncapture cross-sections in the region of $\mathrm{Si}$ are smaller than those in the region of $\mathrm{Fe}$, and because the initial ${ }^{58} \mathrm{Fe}$ abundance is very low. That the ${ }^{30} \mathrm{Si} /{ }^{28} \mathrm{Si}$ ratio increases with a decrease in the metallicity is one piece of evidence for an origin of $\mathrm{SiC}$ grains of type $\mathrm{Y}$ and $\mathrm{Z}$ in AGB stars of metallicity lower than solar (Hoppe et al. 1997; Amari et al. 2001a).

Finally, we show the peculiar case of the ${ }^{50} \mathrm{Ti} /{ }^{48} \mathrm{Ti}$ ratio. Because ${ }^{50} \mathrm{Ti}$ is neutron magic $(N=28)$, its neutron-capture cross section is more than a factor of 10 smaller than those of the other $\mathrm{Ti}$ isotopes. This makes the ${ }^{50} \mathrm{Ti} /{ }^{48} \mathrm{Ti}$ ratio a unique case among the isotopic ratios below $\mathrm{Ni}$, being sensitive to the neutron flux in the ${ }^{13} \mathrm{C}$ pocket. Enhancements in this ratio can reach up to a factor of two and the maximum corresponds to models of mass $3-4 M_{\odot}$ because in this mass range the ${ }^{13} \mathrm{C}$ neutron source is active.

In the top panel of Figures 9-11 we show the final surface composition for elements lighter than $\mathrm{Fe}$ for a selection of stellar evolutionary sequences. The figure illustrates that lowmass stars with $M \leqslant 3 M_{\odot}$ produce substantial $\mathrm{C}, \mathrm{N}$, and $\mathrm{F}$ and some $\mathrm{Ne}$ and $\mathrm{Na}$, where production increases with decreasing metallicity (e.g., Karakas 2010; Cristallo et al. 2011). This is easily understood by examination of Figure 1, which shows that the lowest metallicity $Z=0.007$ dredge up more intershell material at a given mass. The $\mathrm{H}$ and $\mathrm{He}$ intershells of these models are also hotter, owing to a lower opacity. The 

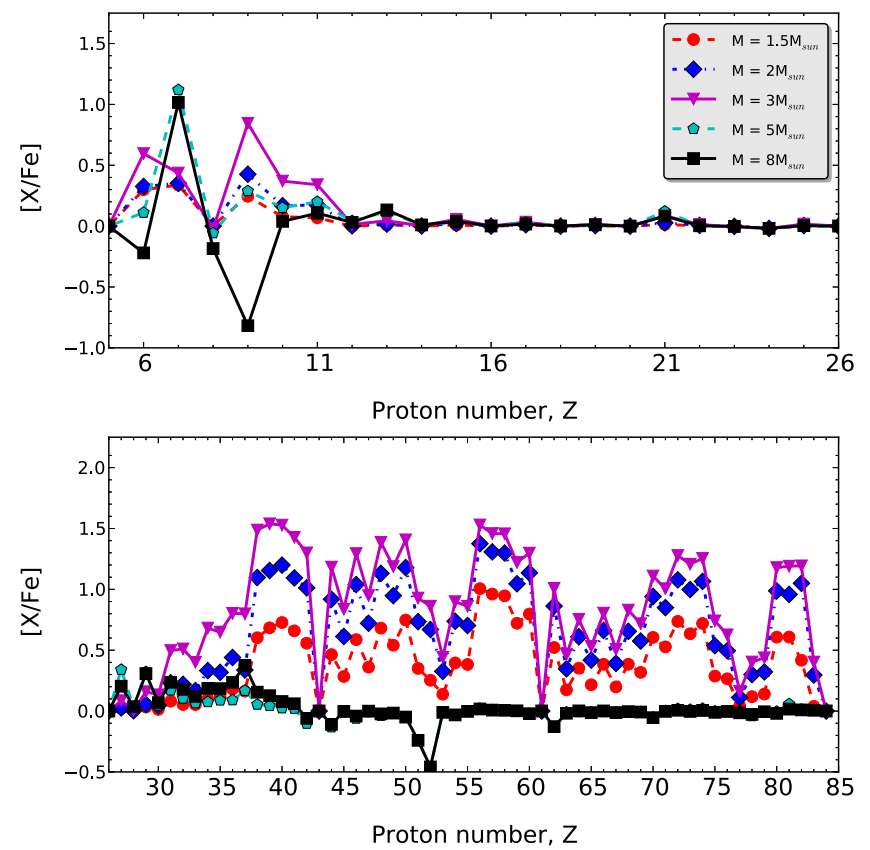

Figure 9. Final elemental surface abundance (in $[\mathrm{X} / \mathrm{Fe}]$ ) for a selection of evolutionary models with a solar metallicity, $Z=0.014$. The masses shown in the figure are indicated in the legend. The top panel shows elements up to the iron peak and the lower panel elements heavier than iron.

intermediate-mass stars $\left(M>3 M_{\odot}\right)$ show substantial $\mathrm{N}$ production and varying degrees of $\mathrm{C}, \mathrm{O}$, and $\mathrm{F}$ destruction. The lowest metallicity $Z=0.007$ intermediate-mass stars also show a small production of $\mathrm{Al}$, where it is well known that the $\mathrm{Mg}-\mathrm{Al}$ chain is more effective at lower metallicity owing to hotter HBB temperatures (e.g., Ventura et al. 2011). For the intermediate-mass elements between $\mathrm{Si}$ and Fe there are almost no changes in the elemental abundance as a consequence of AGB nucleosynthesis (e.g., Cristallo et al. 2009, 2011; Karakas et al. 2009, 2012; Shingles \& Karakas 2013), although there is a small production of $\mathrm{P}$ and $\mathrm{Sc}$ (e.g., at the level of $[\mathrm{Sc} / \mathrm{Fe}] \lesssim$ 0.3 ) as a result of neutron captures (Smith \& Lambert 1989).

\subsection{Elements Heavier Than Iron}

In the lower panel of Figures 9-11 we show the final surface composition for elements heavier than Fe. For the metallicities in our study, intermediate-mass models with masses above $4.5 M_{\odot}$ without ${ }^{13} \mathrm{C}$ pockets show little production of $s$-process elements. This is not the case for lower metallicities $Z \leqslant 0.001$ (Lugaro et al. 2012; Fishlock et al. 2014; Shingles et al. 2015). Some production around the first $s$-process peak at $\mathrm{Rb}$ (number of protons $z=37$ ) occurs, although it is generally $[\mathrm{Rb} / \mathrm{Fe}] \lesssim$ 0.5 for all solar metallicity models, even when a ${ }^{13} \mathrm{C}$ pocket is included. The intermediate-mass models of $Z=0.014$ predict lower $\mathrm{Rb}$ enrichments than models of the same mass and similar metallicity $(Z=0.02,[\mathrm{Fe} / \mathrm{H}]=+0.14)$ from Karakas et al. (2012), except for the $5 M_{\odot}$ case. This is because here we are using an updated ${ }^{22} \mathrm{Ne}(\alpha, \mathrm{n}){ }^{25} \mathrm{Mg}$ reaction from Iliadis et al. (2010), whereas in Karakas et al. (2012) we were using the faster NACRE rate (Angulo et al. 1999). Some production of the elements between $\mathrm{Fe}$ and $\mathrm{Sr}$ occurs, which is typically associated with the weak $s$-process in massive stars, e.g., $\mathrm{Ga}$ $(z=31)$ and $\mathrm{Ge}(z=32)$, as well as peaks at $\mathrm{Co}(z=27)$ and $\mathrm{Cu}(z=29)$.
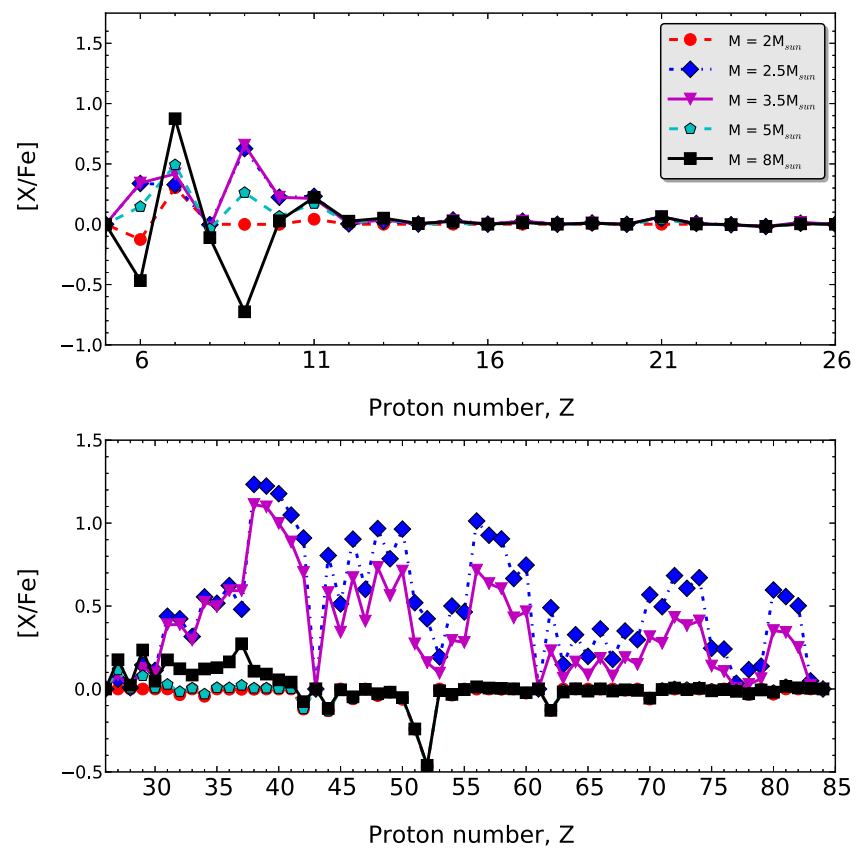

Figure 10. Same as Figure 9 for a selection of $Z=0.03$ models.
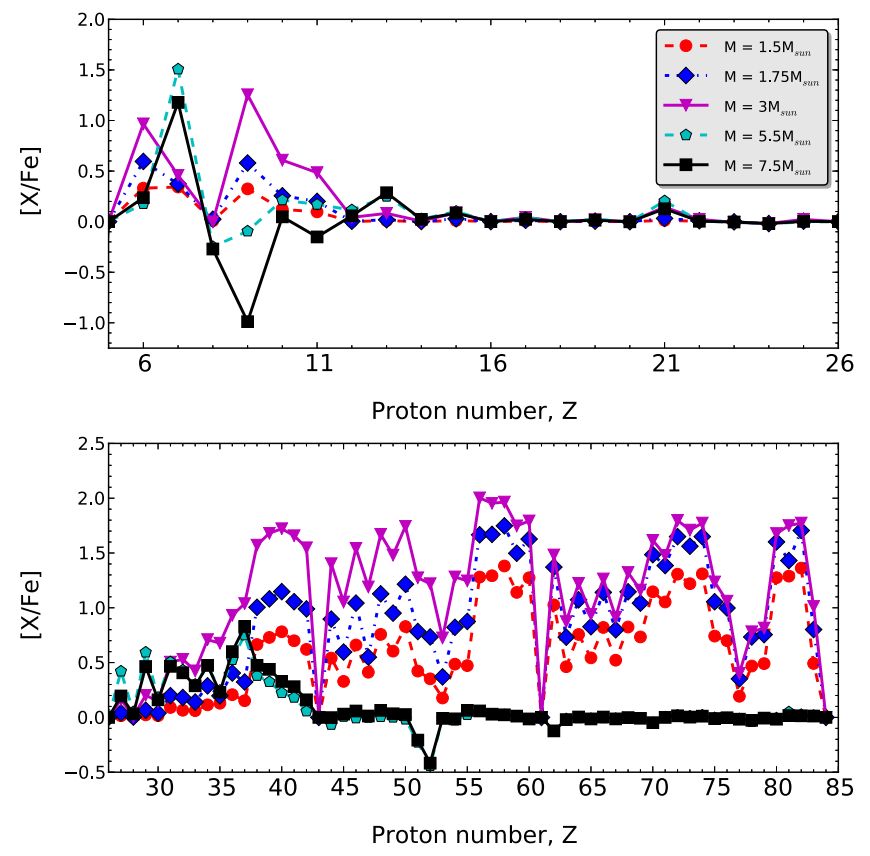

Figure 11. Same as Figure 9 for a selection of $Z=0.007$ models.

In the low-mass models that include ${ }^{13} \mathrm{C}$ pockets the $s$ process production and distribution is strongly dependent on the initial stellar metallicity, as discussed in Busso et al. (2001). Here we focus on metallicities near solar, where production is dominated by elements at the first $s$-process peak, $\mathrm{Sr}, \mathrm{Y}$, and $\mathrm{Zr}$ $(z=38,39,40)$, and the second peak, at $\mathrm{Ba}, \mathrm{La}$, and $\mathrm{Ce}$ $(z=56,57,58)$. This is especially the case for solar and super solar metallicities, as illustrated in Figures 9 and 10. In the lower metallicity $Z=0.007$ we see a noticeable shift in the predicted distribution of $s$-process elements with more secondpeak elements made and a stronger production of $\mathrm{Pb}(z=82)$.

The shift in the $s$-process distribution can be quantified by calculating the $s$-process relative indicators: [hs/ls] and 
$[\mathrm{Pb} / \mathrm{hs}]$, where "ls" stands for light $s$-process elements (Y, Sr, $\mathrm{Zr}$ ) and "hs" for heavy s-process elements (Ba, La, Ce). The $[\mathrm{Rb} / \mathrm{Zr}]$ ratio provides further information on the neutron density in the He-shell. We do not provide [hs $/ \mathrm{Fe}]$ and $[\mathrm{hs} / \mathrm{Fe}]$ in the surface abundance data files, owing to the fact that there are a number of different choices of the elements included in the definition in the literature (Bisterzo et al. 2010; Cristallo et al. 2011; Lugaro et al. 2012). We leave it to readers to calculate these indicators as they choose. However, in the yield tables we provide the $[\mathrm{Rb} / \mathrm{Zr}],[\mathrm{ls} / \mathrm{Fe}],[\mathrm{hs} / \mathrm{Fe}],[\mathrm{hs} / \mathrm{ls}]$, and $[\mathrm{Pb} / \mathrm{ls}]$ ratios, calculated from the integrated yields for each $(M$, $Z$ ) combination, using the elements listed above (Lugaro et al. 2012).

We come back to demonstrating how these ratios vary with mass and metallicity in Section 4 , but here we discuss an illustrative example using the $3 M_{\odot}$ models. The advantage of using the $s$-process relative indicators is that they are largely free of the uncertainties related to the stellar modeling, such as the mass-loss rate and the TDU efficiency (Lugaro et al. 2012). This is important because we see from Figure 1 that the amount of material dredged to the surface increases as a function of decreasing metallicity for models around $3 M_{\odot}$. The [hs $\left./ \mathrm{ls}\right]$ ratios are -0.238 from the $Z=0.03$ model, -0.026 from the solar metallicity model, and +0.320 from the $Z=0.007$ model. This shows the shift from an $s$-process distribution dominated by the first peak in super solar metallicities stars of $[\mathrm{Fe} /$ $\mathrm{H}]=+0.3$, to a distribution dominated by the second peak in stars of $[\mathrm{Fe} / \mathrm{H}]=-0.3$. The distribution in the solar metallicity stars is fairly balanced between the first and second peaks. Furthermore, the $[\mathrm{Pb} / \mathrm{hs}]$ ratio increases from $-0.389,-0.279$, and -0.198 in the $Z=0.03,0.014$, and $Z=0.007$ models, respectively, showing an increase in the $\mathrm{Pb}$ production relative to the second peak.

\section{STELLAR YIELDS}

Stellar yields are an essential ingredient for theoretical calculations of Galactic chemical evolution (Tinsley 1980; Romano et al. 2010; Nomoto et al. 2013; Karakas \& Lattanzio 2014; Mollá et al. 2015). To compute the yields, we integrate the mass lost from the model star during the entire stellar lifetime according to

$$
M_{k}=\int_{0}^{\tau} X(k) \frac{d M}{d t} d t
$$

where $M_{k}$ is the yield of species $k$ (in solar masses), $d M / d t$ is the current mass-loss rate $\left(M_{\odot} \mathrm{yr}^{-1}\right), X(k)$ refers to the current mass fraction of species $k$ at the surface, and $\tau$ is the lifetime of the stellar model. The yield as expressed in Equation (1) is the amount of each element expelled into the interstellar medium over the stellar lifetime (in $M_{\odot}$ ) and is always positive. In Table 7 we show the first few lines of the yield tables for the $3.5 M_{\odot}, Z=0.03$ model as an example. In Figures $12-14$ we show the stellar yields plotted against the initial stellar mass for a selection of elements. In each figure we illustrate the yield and the yield weighted by the Salpeter initial mass function (IMF). We have chosen the Salpeter IMF for simplicity to assess the impact of yields from low-mass AGB stars relative to those from intermediate-mass AGB stars of initial mass over $\approx 4 M_{\odot}$.

In Table 5 we present the stellar yields of Li from models with HBB. If column 5 is subtracted from column 6 , we obtain the net yield of lithium, which provides an indication if the element is produced or destroyed over the star's lifetime. Interestingly, all the net yields of Li from the metal-rich models of $Z=0.03$ are positive, even for the most massive AGB models with strong HBB. This is in contrast to the solar metallicity and lower metallicity $Z=0.007$ models, which only show positive net yields for masses near the minimum mass for HBB $\left(\approx 4.5 M_{\odot}\right)$. In more massive $\mathrm{AGB}$ stars of solar metallicity and lower, Li production peaks early on before much mass is lost from the star. By the time the superwind begins, the star has exhausted its supply of ${ }^{3} \mathrm{He}$ in the envelope and $\mathrm{HBB}$ results in a efficient destruction of $\mathrm{Li}$.

Travaglio et al. (2001) explored the Galactic chemical evolution of Li using yields from intermediate-mass AGB stars similar to those calculated here. The results were that intermediate-mass AGB stars do not play a role in the chemical evolution of lithium in the Galaxy. The mass-loss rates of intermediate-mass stars are uncertain and a stronger mass-loss rate that removes the envelope more quickly may well change this conclusion. Indeed, Prantzos (2012) concludes that a significant fraction of $\mathrm{Li}$ must be produced in low and intermediate mass. Perhaps thermohaline mixing and rotation play a role in the shaping the yields of $\mathrm{Li}$ as they do for ${ }^{3} \mathrm{He}$ (Lagarde et al. 2011).

For the solar metallicity models, the IMF-weighted yield of $\mathrm{N}$ peaks at $\approx 1.5 M_{\odot}$ and at $5 M_{\odot}$, where the low-mass component derives from the FDU and is secondary, and the $5 M_{\odot}$ component derives from HBB and is a mix of primary and second nitrogen. For $\mathrm{Rb}$, the IMF-weighted yields peak at $4 M_{\odot}$; this is because the $4 M_{\odot}$ has both a ${ }^{13} \mathrm{C}$ pocket and a burst of neutrons from the ${ }^{22} \mathrm{Ne}(\alpha, \mathrm{n}){ }^{25} \mathrm{Mg}$ reaction. Models with the ${ }^{22} \mathrm{Ne}(\alpha, \mathrm{n}){ }^{25} \mathrm{Mg}$ reaction alone as a neutron source do not produce enough $s$-process elements, including $\mathrm{Rb}$, to compete with production from the ${ }^{13} \mathrm{C}(\alpha, \mathrm{n}){ }^{16} \mathrm{O}$ reaction in the lower mass stars. For the elements heavier than Rb, AGB stars between about $1.5-3 M_{\odot}$ dominate production as expected (e.g., Busso et al. 2001). The peak occurs at $\sim 2 M_{\odot}$ for Ba, La, and $\mathrm{Pb}$, which reflects the IMF and the TDU efficiency, and at $3 M_{\odot}$ for $\mathrm{Sr}$ and $\mathrm{Y}$, which reflects the contribution of the ${ }^{22} \mathrm{Ne}$ $(\alpha, \mathrm{n})^{25} \mathrm{Mg}$ neutron source to these elements. Also for $\mathrm{F}$ the peak is $3 M_{\odot}$ owing to the fact that the ${ }^{15} \mathrm{~N}(\alpha, \gamma){ }^{19} \mathrm{~F}$ reaction that produces $\mathrm{F}$ in the intershell is most efficient at that mass, as discussed earlier in Section 3.

For the metal-rich $Z=0.03$ models illustrated in Figure 13, the IMF-weighted yields of $s$-process elements also show a strong peak in the models that experience TDU and ${ }^{13} \mathrm{C}$ pockets. The peak for all $s$-process elements is observed at the lowest mass that becomes $\mathrm{C}$-rich, which is $2.5 \mathrm{M}_{\odot}$ according to our assumptions, which reflects the shape of the IMF and also the fact that at this metallicity the effect of the ${ }^{22} \mathrm{Ne}$ neutron source on elements such as $\mathrm{Sr}$ and $\mathrm{Y}$ is marginal. The yields of light $s$-process elements ( $\mathrm{Sr}, \mathrm{Y}$ ) are higher than the yields of heavy $s$-process elements $(\mathrm{Ba}, \mathrm{La})$. The yields of $\mathrm{Pb}$ are lower than in the models of lower metallicity. We conclude that AGB models with metallicity $Z=0.03$ do not contribute greatly to the chemical enrichment of elements heavier than La, although a full chemical evolution model is needed to test this. The weighted yields of lighter elements $\mathrm{C}$ and $\mathrm{F}$ show a strong increase toward lower mass due to the IMF, while the yield of $\mathrm{N}$ is relatively flat with initial mass.

The $Z=0.007$ models shown in Figure 14 show a strong production of all elements. Here the IMF-weighted $\mathrm{N}$ yields 
Table 7

Example of the Yield Tables Available

\begin{tabular}{|c|c|c|c|c|c|c|}
\hline \multicolumn{7}{|c|}{ \# Initial mass $=3.500, Z=0.0300, Y=0.300, M_{\text {mix }}=1.00 \mathrm{E}-03$} \\
\hline$\# \mathrm{El}$ & $Z$ & $\log e(X)$ & {$[\mathrm{X} / \mathrm{H}]$} & {$[\mathrm{X} / \mathrm{Fe}]$} & $X(i)$ & $\operatorname{Mass}(i)$ \\
\hline $\mathrm{p}$ & 1 & 12.000000 & 0.000000 & 0.000000 & $6.35230 \mathrm{E}-01$ & $1.76149 \mathrm{E}+00$ \\
\hline he & 2 & 11.119857 & 0.189857 & -0.165502 & 3.32440E-01 & $9.21855 \mathrm{E}-01$ \\
\hline c & 6 & 9.147092 & 0.677092 & 0.321733 & 1.06204E-02 & 2.94504E-02 \\
\hline $\mathrm{n}$ & 7 & 8.643233 & 0.773233 & 0.417875 & 3.88194E-03 & $1.07646 \mathrm{E}-02$ \\
\hline o & 8 & 9.057708 & 0.327708 & -0.027650 & $1.15152 \mathrm{E}-02$ & $3.19316 \mathrm{E}-02$ \\
\hline$f$ & 9 & 5.442862 & 0.982862 & 0.627503 & 3.31925E-06 & $9.20429 \mathrm{E}-06$ \\
\hline
\end{tabular}

Note. We show the first few lines of the $3.5 M_{\odot}, Z=0.03$ model yield table.

(This table is available in its entirety in FITS format.)
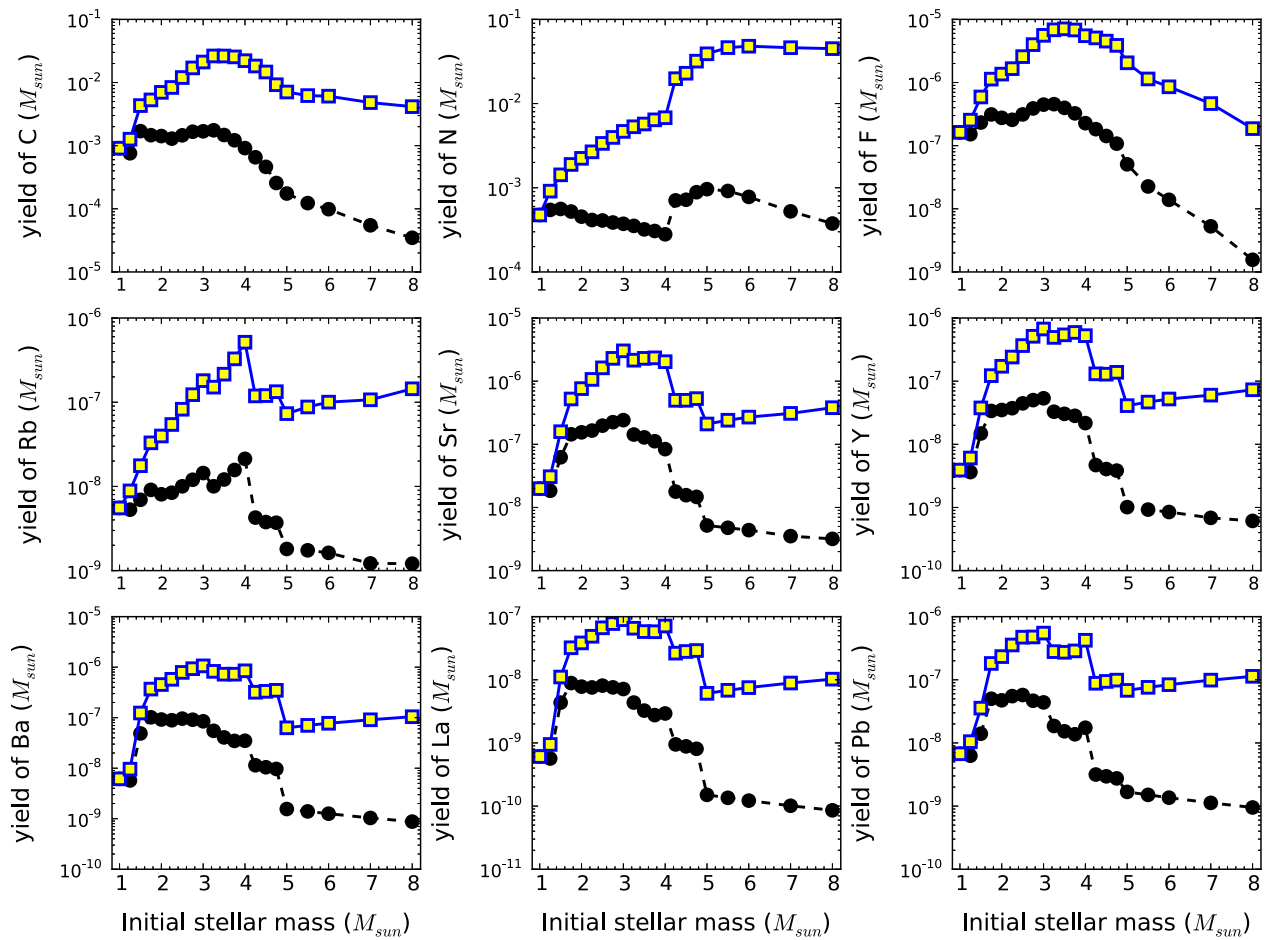

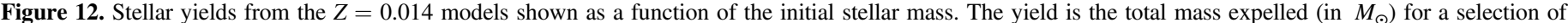
elements (blue and yellow squares) compared to the yields weighted by the Salpeter IMF (black filled circles).

show a strong preference for intermediate-mass AGB production owing to the efficient $\mathrm{HBB}$ found for models above 4.5 $M_{\odot}$. The effect of HBB is also visible in the yields of $\mathrm{C}$ and $\mathrm{F}$, as a decrease in models with $\mathrm{HBB}$. The yield of $\mathrm{Rb}$ peaks at $4 M_{\odot}$ as it did at solar metallicity and for the same reasons. Elements heavier than $\mathrm{Rb}$ are again dominated by the low-mass AGB stars that experience TDU and ${ }^{13} \mathrm{C}$ pockets. As in $Z=0.014$ case, the $\mathrm{Sr}$ and $\mathrm{Y}$ preference for $\sim 3 M_{\odot}$ models is driven by the contribution of the ${ }^{22} \mathrm{Ne}$ neutron source at this mass, while the $\mathrm{Ba}, \mathrm{La}$, and $\mathrm{Pb}$ preference for $\sim 2 M_{\odot}$ models is driven by the balance between the IMF and the TDU efficiency.

In Figures 15-17 we show how the $s$-process indicators [Rb/ $\mathrm{Zr}$, [ls/Fe], [hs/ls], and [Pb/hs] vary as a function of stellar mass for the three metallicities considered in this study. We also show the IMF-weighted $s$-process indicators. The weighting removes most of the noise and is indicative of what is expected in a Galactic chemical evolution model. In Figure 15 we also show the range of ratios expected from predictions when varying the size of $M_{\mathrm{mix}}$, which controls the size of the
${ }^{13} \mathrm{C}$ pocket in our calculations. We only plot this for the solarmetallicity models because we have the most extensive grid for different values of $M_{\text {mix }}$ (Table 3 ). The large ranges indicate how this parameter is still the major uncertainty affecting the $s$ process in AGB stars; however, it cannot be considered as a real error bar, but rather an illustration of the difficulty of quantifying this uncertainty.

In more detail, using the [ls/Fe] ratio as a proxy of the absolute abundance produced (i.e., the yields), it can be seen that variations are very large when $M_{\text {mix }}$ is varied in the case of the 2 and $3 M_{\odot}$ stars. In these models we varied $M_{\text {mix }}$ down to zero, in which case there are almost no neutrons. The error bars cover the observations of AGB stars that range from 0 to $1 \mathrm{dex}$ (Busso et al. 2001; Abia et al. 2002); however, we need to be cautious before reaching conclusions on the size and variation of $M_{\mathrm{mix}}$, because low [ls/Fe] values can also be explained by stars of lower mass, as shown in the figure.

The models mostly affected by the uncertainty on $M_{\text {mix }}$ are those at the transition between the low- and the intermediate- 

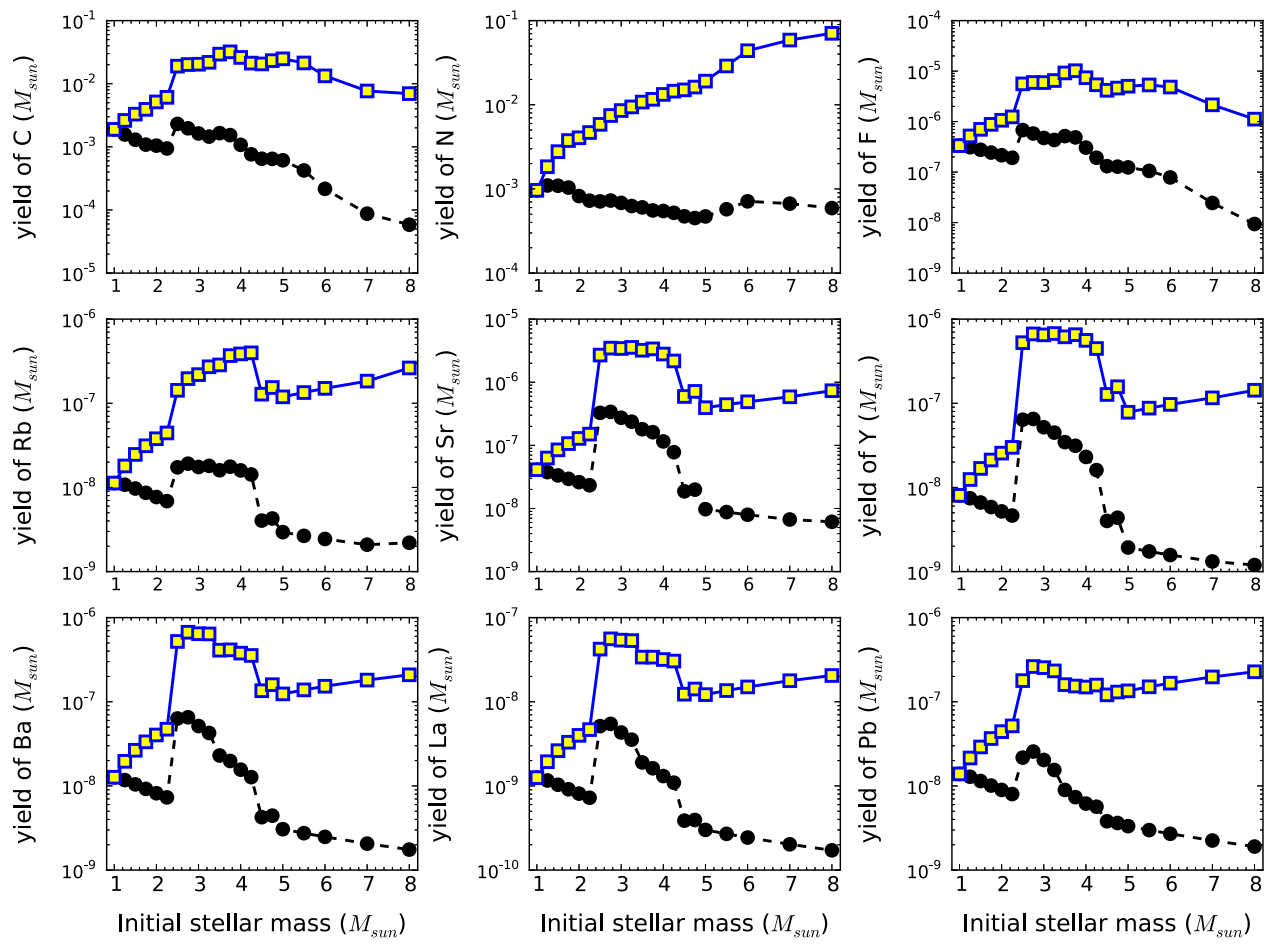

Figure 13. Same as Figure 12 but for the $Z=0.03$ models.
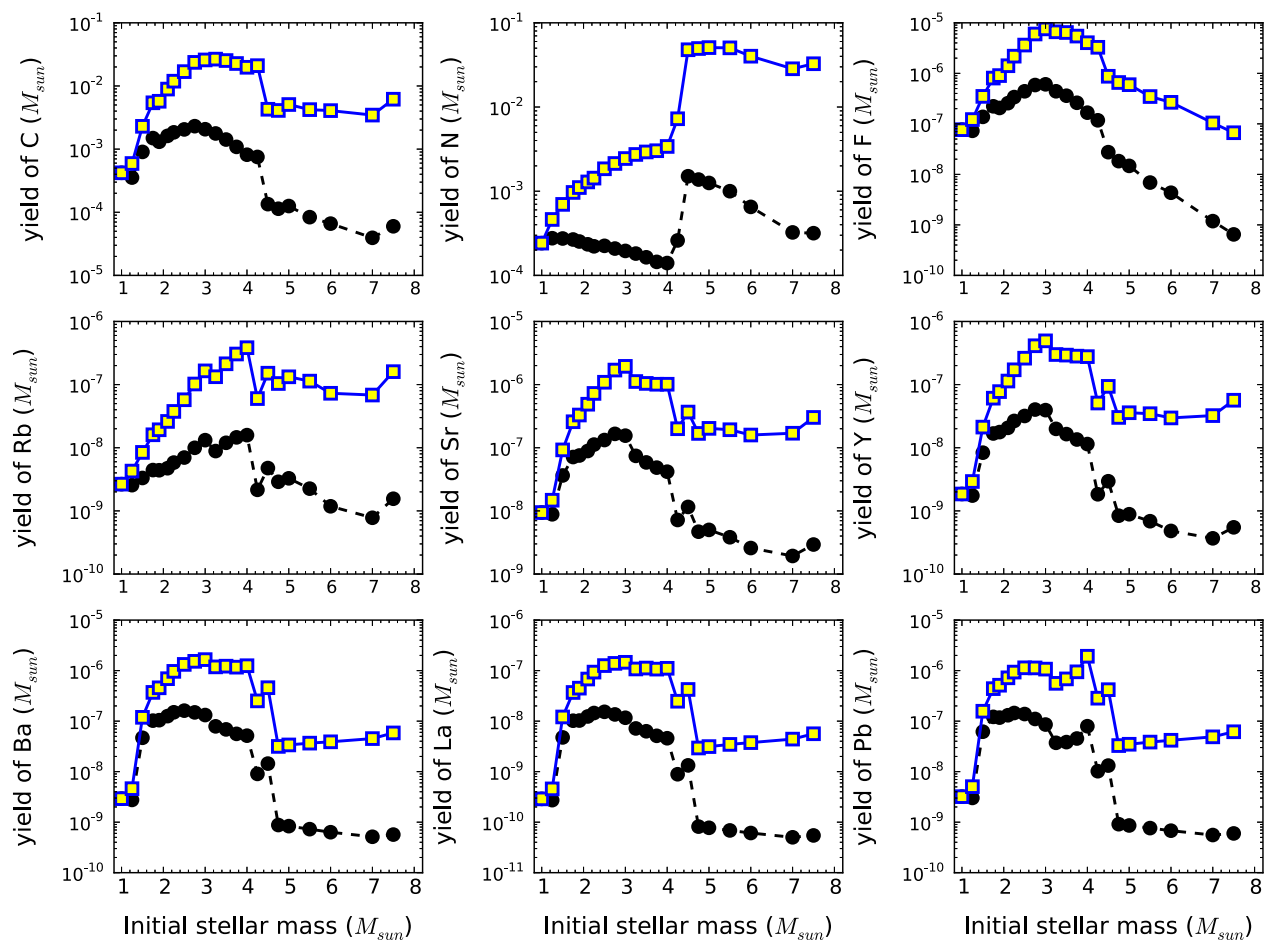

Figure 14. Same as Figure 12 but for the $Z=0.007$ models.

mass regime, i.e., between 4 and $5 M_{\odot}$ in the figure. It is difficult to identify clear observational constraints attributable to this mass range. Moreover, model uncertainties will play a role in determining exactly how $M_{\text {mix }}$ should scale down as the intershell mass becomes smaller with evolution. Also, HBB can be partially activated during the AGB in models of this mass, which means that the effect of the hot dredge up (Goriely \& Siess 2004) may appear or disappear. For these models, we experiment with a range of plausible $M_{\text {mix }}$ values. For higher masses, the uncertainty becomes smaller given the observational evidence (García-Hernández et al. 2013) for the absence of ${ }^{13} \mathrm{C}$ pockets.

The $[\mathrm{Rb} / \mathrm{Zr}]$ ratio is very sensitive to the neutron source active in the He-intershell: negative values indicate that the ${ }^{13} \mathrm{C}(\alpha, \mathrm{n}){ }^{16} \mathrm{O}$ neutron source reaction is dominant while positive values indicate the ${ }^{22} \mathrm{Ne}$ neutron source. This comes about 

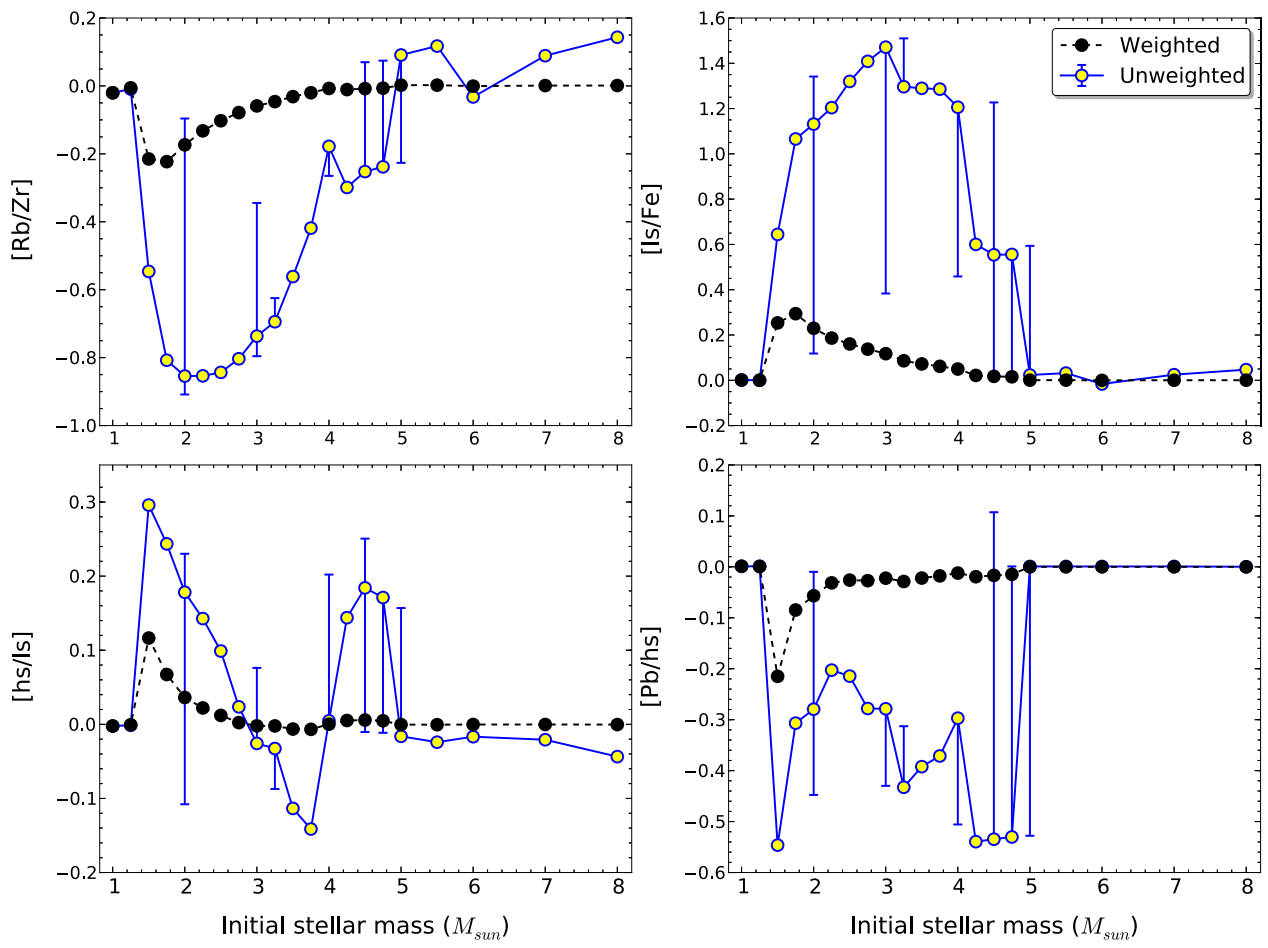

Figure 15. $s$-process indicators $[\mathrm{Rb} / \mathrm{Zr}],[\mathrm{ls} / \mathrm{Fe}],[\mathrm{hs} / \mathrm{ls}]$, and $[\mathrm{Pb} / \mathrm{hs}]$ as a function of initial stellar mass (blue and yellow circles) for the $Z=0.014$ models. The ratios are calculated from the integrated yield abundances, not from the final surface abundances. We varied the mass of $M_{\text {mix }}$ as indicated in Table 3 , with results shown as error bars on the blue line. These give some indication of the range of behavior as a function of stellar mass and ${ }^{13} \mathrm{C}$ pocket size for models of this metallicity. The black filled circles show the $s$-process indicators weighted by the Salpeter IMF.
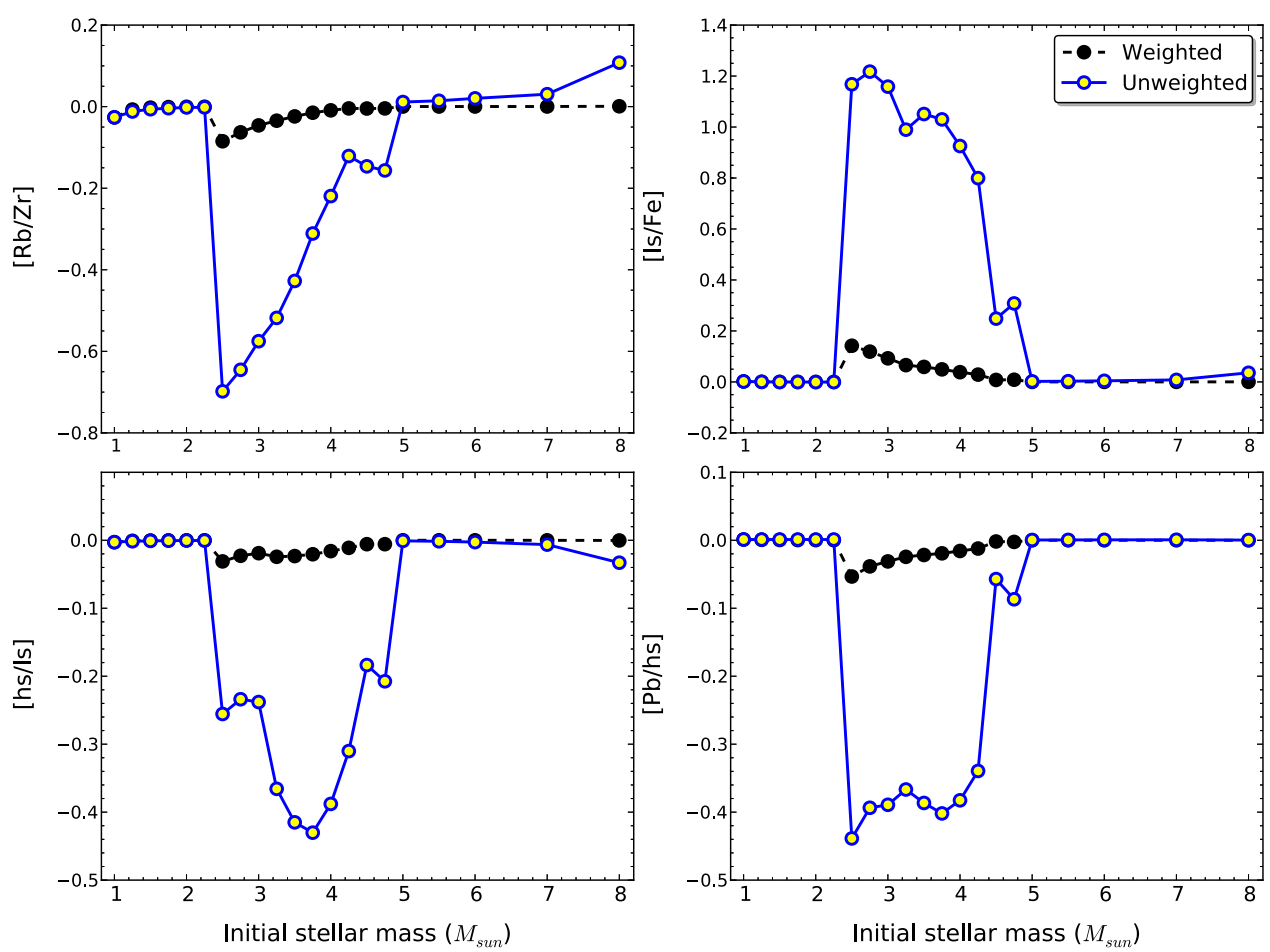

Figure 16. Same as Figure 15 but for the $Z=0.03$ models. Here we only show s-process indicators for our standard choice of $M_{\text {mix }}$.

because the density of neutrons released by the ${ }^{13} \mathrm{C}$ reaction are lower $\left(n_{\mathrm{n}} \lesssim 10^{8} \mathrm{n} \mathrm{cm}^{-3}\right)$ than the peak neutron density from the ${ }^{22} \mathrm{Ne}$ neutron source $\left(n_{\mathrm{n}} \approx 10^{13} \mathrm{n} \mathrm{cm}^{-3}\right.$, which allows branching points to open to produce Rb, e.g., van Raai et al. 2012; Fishlock et al. 2014). We only see positive values for the intermediate-mass models above $4 M_{\odot}$ (unless a ${ }^{13} \mathrm{C}$ pocket is included), with the largest $[\mathrm{Rb} / \mathrm{Zr}]$ obtained in the lowest metallicity models. 

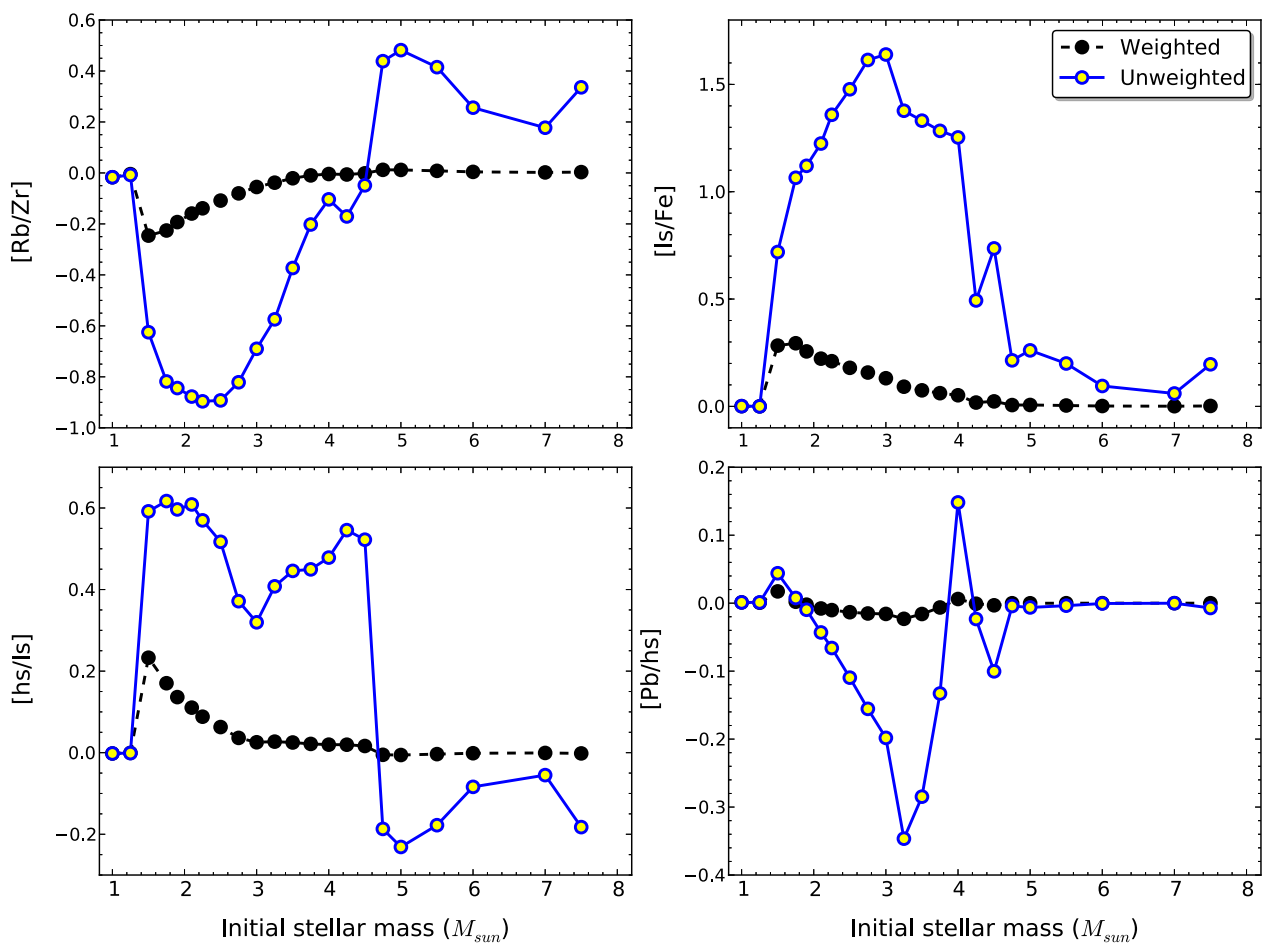

Figure 17. Same as Figure 16 but for the $Z=0.007$ models.

\section{COMPARISON WITH OTHER YIELD SETS}

Here we compare the results from the $1.5,3$, and $6 M_{\odot}$ models to models of the same mass and similar $Z$ from the FRUITY database (Cristallo et al. 2011, 2015). We also compare our $5 M_{\odot}, Z=0.014$ model and yields to models of the same mass from the NuGrid database (Pignatari et al. 2013). We note that a good agreement between yields does not mean that the yields are free of uncertainties; indeed, such agreement could be coincidental. Comparing to observations is the most reliable method to verify the accuracy of the predictions.

Cristallo et al. (2015) also use $Z=0.014$ for the solar metallicity, which makes a direct comparison straightforward. There are no AGB models published with $Z=0.03$, although there are slightly super solar metallicity $(Z=0.02)$ models available from the FRUITY database. FRUITY include models with $Z=0.008$ or $Z=0.006$, and our lower metallicity $Z=0.007$ models are in the middle of that range.

For comparison it is useful to keep in mind the differences between the FRUITY models and those presented here. In particular, we assume no mass loss on the RGB and Vassiliadis \& Wood (1993) mass loss on the AGB. Cristallo et al. (2015) adopt a Reimer's mass-loss rate with $\eta=0.4$ for the pre-AGB phase and then use the formulae from Straniero et al. (2006) for the AGB. In addition to differences in the mass-loss rates, there are also significant differences in our treatment of convection and convective borders, which were discussed in Section 2.2. Differences in the nuclear physics input can also sometimes play a role.

In Figure 18 we compare the results for the $3 M_{\odot}, Z=0.014$ models. The agreement between the light elements $\mathrm{C}, \mathrm{N}$, and $\mathrm{O}$ is reasonably good, while our model produces more $\mathrm{F}, \mathrm{Ne}$, and $\mathrm{Na}$, and more elements heavier than Fe. The $s$-process distribution is very similar with the $[\mathrm{Ba} / \mathrm{Sr}]$ and $[\mathrm{Ba} / \mathrm{Pb}]$ ratios the same to within 0.15 dex. Here we are comparing to our $3 M_{\odot}, Z=0.014$
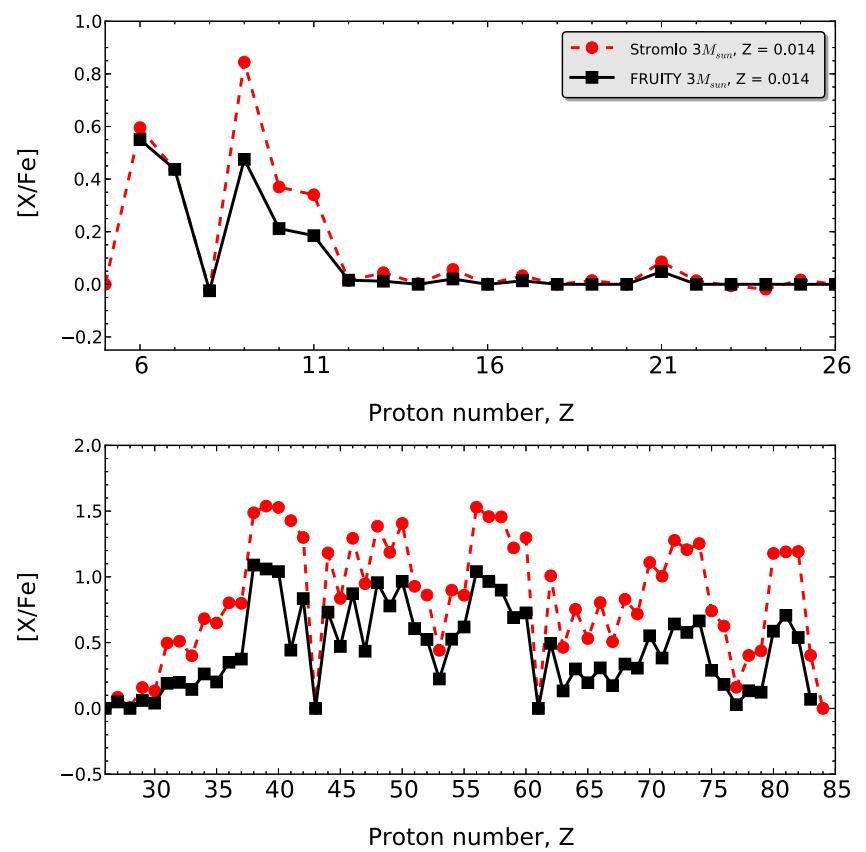

Figure 18. Comparison between the nucleosynthesis results from $3 M_{\odot}$, $Z=0.014$ models. We are showing the final elemental surface composition (in $[\mathrm{X} / \mathrm{Fe}])$ for each case. The Stromlo model refers to the model presented here. We have set $[\mathrm{Tc}, \mathrm{Pm} / \mathrm{Fe}]=0$ in the figures (Tc and $\mathrm{Pm}$ have proton numbers $z=43$ and $z=61$, respectively), whereas in reality these abundance ratios are not defined because these elements are radioactive and have a zero solar abundance.

model with $M_{\text {mix }}=2 \times 10^{-3} M_{\odot}$, noting that the models with smaller partially mixed zones produce less heavy elements, and are closer to the FRUITY model. However, even the model with the smallest $M_{\text {mix }}=1 \times 10^{-4} M_{\odot}$ still produces $[\mathrm{F} / \mathrm{Fe}]=0.74$, which is higher than the FRUITY predictions for this mass 

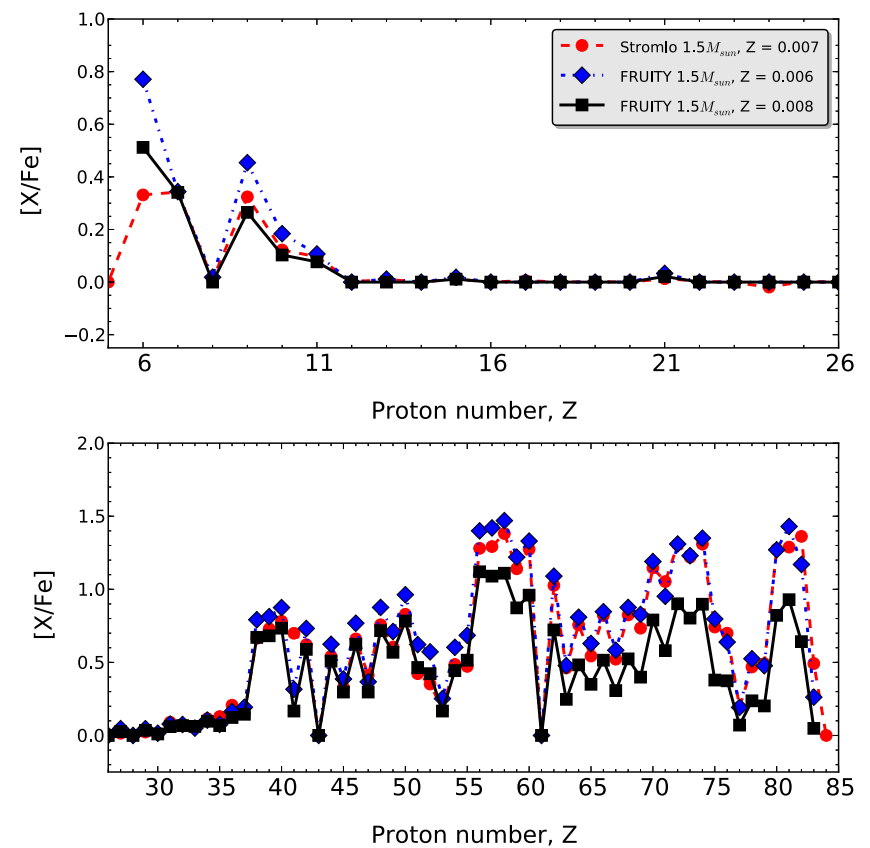

Figure 19. Comparison between the nucleosynthesis results from $1.5 M_{\odot}$, $Z=0.007$ Stromlo model, and the $1.5 M_{\odot}$ models from FRUITY with $Z=0.006$ and $Z=0.008$. Results are shown for the final elemental surface composition.

$([\mathrm{F} / \mathrm{Fe}]=0.475)$, although the agreement with $\mathrm{Ne}$ and $\mathrm{Na}$ improves. This suggests that our $\mathrm{F}$ abundances are higher owing to differences in the nuclear network.

Comparing the amount of TDU, the Stromlo $3 M_{\odot}$ and $6 M_{\odot}$, $Z=0.014$ models dredge up roughly the same amount of material $\left(\approx 0.1 M_{\odot}\right)$, as shown in Figure 1 . The $6 M_{\odot}$, $Z=0.007$ also dredges up about $0.1 M_{\odot}$. The FRUITY $3 M_{\odot}$ of solar composition dredges up $0.06 M_{\odot}$, roughly $60 \%$ less material than the Stromlo model of the same mass and composition. This difference explains the higher absolute F, $\mathrm{Na}$, and $s$-process abundances that we see in Figure 18. The Stromlo model dredges up more material because it experiences more TPs and deeper TDU. The FRUITY model has 17 TPs compared to 28 in the Stromlo case. While the FRUITY model experiences deeper TDU sooner than the Stromlo case, the Stromlo model has a higher peak TDU efficiency as measured using the parameter $\lambda$ (e.g., $\lambda_{\max } \approx 0.8$ compared to $\lambda_{\max } \approx 0.6$ from the FRUITY model). Interestingly, the $\mathrm{C}$ abundances are similar in the $3 M_{\odot}$ models, even though the Stromlo model dredges up $60 \%$ more He-shell. That the surface $\mathrm{C}$ abundances are coincidently the same implies that the FRUITY models have a higher $\mathrm{C}$ abundance in their intershell. The cause probably lies in the choice of triple- $\alpha$ reaction rate: FRUITY use the NACRE rate, which is $10 \%$ faster (at $T=200 \mathrm{MK}$ ) than the rate we use from the JINA reaclib database.

In Figures 19 and 20 we compare the results from our $1.5 M_{\odot}$ and $6 M_{\odot}$ models with $Z=0.007$ to models of similar metallicity from the FRUITY database. For the $1.5 M_{\odot}$ case, the two FRUITY models of $Z=0.006$ and $Z=0.008$ predict $\mathrm{C}$ and $\mathrm{F}$ abundances that increase with decreasing $Z$. The Stromlo $1.5 M_{\odot}$ model produces less $\mathrm{C}$ than either FRUITY model but similar F, confirming the results for the $3 M_{\odot}$, solar metallicity case. The results for the heavy elements are similar for all three
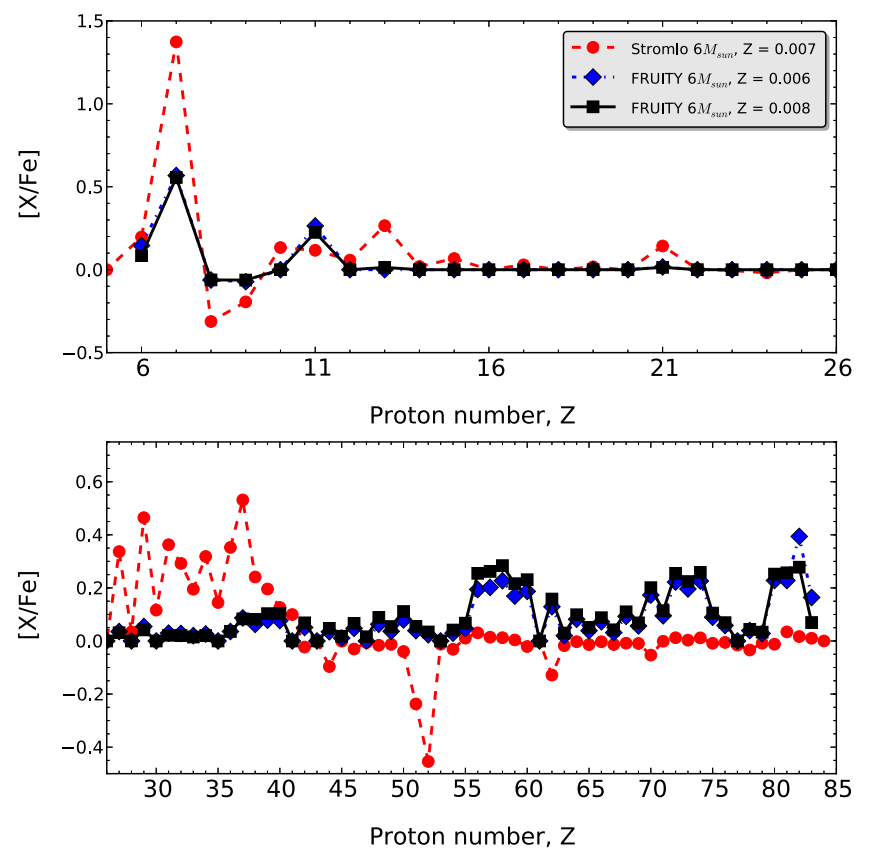

Figure 20. Same as Figure 19 except for the $6 M_{\odot}, Z=0.007$ model.

models, with the Stromlo model lying close to the predictions of the FRUITY $Z=0.006$ model. The main reason for the reasonable agreement between the distribution of abundances shown in Figure 19 is that the models dredge up roughly about the same amount of material. The Stromlo $1.5 M_{\odot}, Z=0.007$ model dredges up $7.6 \times 10^{-3} M_{\odot}$ compared to $\approx 8 \times 10^{-3} M_{\odot}$ and $0.014 M_{\odot}$ from the FRUITY $Z=0.008$ and $Z=0.006$ models, respectively.

The largest discrepancies are found for models of intermediate mass such as the case of the $6 M_{\odot}, Z=0.007$ model illustrated in Figure 20. The Stromlo model has stronger HBB, which is evident from the production of $\mathrm{N}$ and $\mathrm{Al}$ and the destruction of $\mathrm{O}$ and $\mathrm{F}$. On the other hand, there is very little destruction of these elements in the FRUITY $6 M_{\odot}$ case. The discrepancies between the FRUITY models and the Stromlo model continue into the heavy elements. The Stromlo model shows evidence of the ${ }^{22} \mathrm{Ne}(\alpha, \mathrm{n})^{25} \mathrm{Mg}$ neutron source, with a strong production of elements at the first $s$-process peak (the final $[\mathrm{Rb} / \mathrm{Fe}]=0.53$ ) and little $s$-process production beyond that. ${ }^{9}$ In contrast, the FRUITY $6 M_{\odot}$ models show almost no production of light $s$-process elements and instead some production around the $\mathrm{Ba}$ and $\mathrm{Pb}$ peaks, caused by the formation of a small ${ }^{13} \mathrm{C}$ pocket (Straniero et al. 2014; Cristallo et al. 2015). We do not see any indication of the activation of ${ }^{22} \mathrm{Ne}$ in the FRUITY model from the surface composition.

The discrepancies illustrated in Figure 20 can mostly be traced back to the input physics used in the models. The FRUITY $6 M_{\odot}$ models lose considerably more mass before the first TP and experience fewer TPs, as discussed above for the $1.5 M_{\odot}$ and $3 M_{\odot}$ models. Our $6 M_{\odot}, Z=0.007$ model shown in Figure 20 has 64 TPs, roughly 3 times more TPs in the FRUITY models of similar composition. Fewer TPs results in less material dredged to the stellar surface, where the FRUITY models of $6 M_{\odot}, Z=0.006$ and $Z=0.008$ dredge up a factor of 11 to 18 times less than our $6 M_{\odot}, Z=0.007$ model. Less

\footnotetext{
9 The low abundance of Te is because we do not include all stable isotopes of this element; see the discussion in Lugaro et al. (2012).
} 
material from the He-intershell means that there is fewer ${ }^{12} \mathrm{C}$ nuclei in the envelope available to be converted into ${ }^{14} \mathrm{~N}$ by $\mathrm{HBB}$.

The smaller dredge up in the FRUITY models explains why our model has higher absolute enhancements in $s$-process elements. However, this alone does not explain the differences in the $s$-process distributions. Our model shows activation of the ${ }^{22} \mathrm{Ne}(\alpha, \mathrm{n}){ }^{25} \mathrm{Mg}$ neutron source, with a peak in production around $\mathrm{Rb}$. We do not include ${ }^{13} \mathrm{C}$ pockets into the $6 M_{\odot}$ models, so we see no evidence of the ${ }^{13} \mathrm{C}$ neutron source; that is, we have low $\mathrm{Ba}, \mathrm{La}$, and $\mathrm{Pb}$ abundances. In contrast, the FRUITY $6 M_{\odot}$ models only show enhancements in $\mathrm{Ba}$ and heavier elements, indicating that their models develop ${ }^{13} \mathrm{C}$ pockets as a consequence of their treatment of convection and convective borders (as discussed in Cristallo et al. 2015). On the other hand, the signature of the ${ }^{22} \mathrm{Ne}(\alpha, \mathrm{n})^{25} \mathrm{Mg}$ neutron source is not present at the stellar surface. This again can probably be traced back to the different mass-loss law. The ${ }^{22} \mathrm{Ne}(\alpha, \mathrm{n}){ }^{25} \mathrm{Mg}$ neutron source is typically activated more efficiently in the later rather than earlier TPs (opposite to the ${ }^{13} \mathrm{C}$ neutron source, which is activated from the first TDU episode), which explains why the FRUITY models do not show its effect at the stellar surface.

Observations of Galactic AGB stars (García-Hernández et al. 2013) found no evidence for the activation of the ${ }^{13} \mathrm{C}$ neutron source in intermediate-mass stars, when using Tc as a tracer. On the other hand, observations of the brightest intermediatemass AGB stars both in the Galaxy and in the Magellanic Clouds reveal a surface chemistry that is O-rich and $s$-process rich (Wood et al. 1983; García-Hernández et al. 2006, 2009). This is at odds with the model predictions from the FRUITY database which predict a final $[\mathrm{Rb} / \mathrm{Fe}]<0.1$, although the Stromlo models also fail to quantitatively account for the huge $\mathrm{Rb}$ enrichments observed (e.g., Karakas et al. 2012; van Raai et al. 2012). Circumstellar effects have been found to cause large overestimates when determining the abundances of $\mathrm{Rb}$. These effects do not remove the enrichments and in fact bring the model predictions by Karakas et al. (2012) more in line with observations (Zamora et al. 2014).

The other major discrepancy between the intermediate-mass models concerns the strength of HBB. Other stellar evolution codes predict HBB as strong as ours (e.g., Herwig 2004; Weiss $\&$ Ferguson 2009; Pignatari et al. 2013), or stronger in the case of the Full Spectrum of Turbulence models calculated using the ATON code (e.g., Ventura et al. 2013). In comparison to the FRUITY models, the Stromlo code predicts considerably higher temperatures at the base of the envelope during HBB (as also highlighted by Fishlock et al. 2014; Shingles et al. 2015). From Figure 9 from Cristallo et al. (2015), the peak $\mathrm{HBB}$ temperatures in their $6 M_{\odot}, Z=0.014, Z=0.008$ and $Z=0.006$ models are $\log T_{\text {bce }}^{\max } / K \approx 7.25,7.35$, and 7.45 , respectively. ${ }^{10}$ In contrast, our $6 M_{\odot}$ models have maximums of $\log T_{\mathrm{bce}}^{\max } / K=7.85,7.93,7.96$, for metallicities $Z=0.03$, $Z=0.014$, and $Z=0.007$, respectively. Cristallo et al. (2015) explore various reasons why their models experience lower temperatures at the base of the convective envelope but did not identify the cause.

We initially speculated if the higher mass loss experienced by the FRUITY models is the cause. The $6 M_{\odot}, Z=0.014$ model from Cristallo et al. (2015) loses $\approx 1 M_{\odot}$ on the early

\footnotetext{
10 These numbers are approximate and read from the figure. HBB temperatures are not provided on the online FRUITY database.
}

AGB and enters the TP-AGB with a total mass of $5.1 M_{\odot}$, effectively a $5 M_{\odot}$ model star. This is very different to what we find when using the Vassiliadis \& Wood (1993) prescription, where less than $0.1 M_{\odot}$ is lost during the early AGB. The reason for the rapid early AGB mass loss comes down to the calculation of the pulsation period, which determines the rate of mass loss. A detailed comparison between our $6 M_{\odot}$ model and the FRUITY model finds excellent agreement between the radius and luminosity at the beginning of the early AGB and at the start of the TP-AGB. ${ }^{11}$ However, our pulsation periods, as calculated using the period-radius-mass relation from Vassiliadis \& Wood (1993, their Equation (4)), are roughly a factor of 10 lower. Cristallo et al. (2015) calculate the pulsation period using a $M_{\mathrm{k}}-\log P$ relation (e.g., Whitelock et al. 2008), which requires calculation of $M_{\mathrm{k}}$ first from stellar variables.

Going back to the connection between HBB and mass loss, we perform a test calculation and adopt the faster Blöcker (1995) mass-loss rate on the AGB with $\eta=0.4$ in a $6 M_{\odot}$, $Z=0.014$ model in order to strip of the envelope mass quickly. Now the mass loss increases such that we only calculate 10 TPs (e.g., 53 when using Vassiliadis \& Wood 1993). The peak temperature still reaches $\log T_{\mathrm{bce}}^{\max } / K=7.72$, which is roughly a factor of 3 higher than the peak HBB temperature found in the Cristallo et al. model of the same mass and composition. We conclude that while the mass-loss rates found by the FRUITY calculations are higher, the lower HBB temperatures are not caused by their choice of mass loss on the AGB.

The process of HBB in intermediate-mass AGB models explains the observational fact that the most luminous AGB population in the Magellanic Clouds is dominated by O-rich AGB stars, while the less luminous AGB population is dominated by C-rich AGB stars (Wood et al. 1983). Cristallo et al. (2015) note that the effect of HBB may be mimicked by rapid rotation in intermediate-mass AGB stars. At the present time there are no intermediate-mass AGB models with rotation available at the metallicities of the Large Magellanic Cloud or solar metallicity to check if rapid rotation does indeed mimic the signature of HBB.

The brightest O-rich AGB stars in the Magellanic Clouds and Galaxy are also rich in Li (Smith \& Lambert 1990; GarcíaHernández et al. 2013). In Table 5 we show the Li abundances from our models with HBB, which confirms that our intermediate-mass AGB models with HBB become Li-rich (e.g., see also Lattanzio et al. 1997; van Raai et al. 2012). For example, the peak $\mathrm{Li}$ abundances in models of $6 M_{\odot}$ are $\log \epsilon(\mathrm{Li})=4.87, \quad 4.28, \quad 4.42, \quad$ respectively, for $Z=0.03,0.014$, and $Z=0.007$, confirming that the models are both O-rich and super-Li-rich, at least for a while. It would be interesting to test if intermediate-mass AGB models with rotation also become Li-rich.

The NuGrid/MESA collaboration calculated models and yields of low- and intermediate-mass AGB stars (Pignatari et al. 2013). Their grid includes models of $M=1.65,2,3,4$, $5 M_{\odot}$ with $Z=0.01$ and $Z=0.02$. For each $(M, Z)$ combination, stellar evolution model data and detailed yields are provided. Given the discrepancies we found between our intermediate-mass AGB models with $\mathrm{HBB}$ and those from the FRUITY database we compare our results to the NuGrid intermediate-mass $5 M_{\odot}$ AGB models. Our $5 M_{\odot}, Z=0.014$

\footnotetext{
${ }^{11} \mathrm{We}$ also find excellent agreement between our core $\mathrm{H}$ - and He-burning lifetimes to better than $5 \%$.
} 
model is in the middle of NuGrid metallicity range. First, we note that the NuGrid $5 M_{\odot}$ models both experience HBB, with peak temperatures at the base of the envelope of $50 \mathrm{MK}$ and 65 MK, respectively (Tables 6 and 7 from Pignatari et al. 2013). At first glance, our $5 M_{\odot}, Z=0.014$ model would appear to experience stronger $\mathrm{HBB}$, with a peak temperature of $75 \mathrm{MK}$. However, this is likely because the Vassiliadis \& Wood (1993) mass-loss rate is slower and maintains a more massive envelope for longer, compared to the Blöcker (1995) massloss rate used in the MESA calculations. This can be quantified by comparing the number of TPs: our model has 41 TPs compared to the 25 TPs and 22 TPs experienced by the $5 M_{\odot}$ NuGrid models of $Z=0.02$ and $Z=0.01$, respectively. Note that the NuGrid $5 M_{\odot}$ models reach their peak HBB temperature after about 11 TPs. After 11 TPs, the temperature at the base of the envelope of our $5 M_{\odot}$ model is $52 \mathrm{MK}$, comparable to the NuGrid $Z=0.02$ model. This demonstrates that HBB is consistent between our models and the NuGrid/ MESA models.

The Stromlo model dredges up roughly 3 and 4 times as much material as the NuGrid $Z=0.02$ and $Z=0.01$ models, respectively. This, combined with envelope burning extended over more TPs, results in higher yields of most elements, except for carbon, which is destroyed in the Stromlo models. The yield of $\mathrm{Rb}$ is the same to within $5 \%$ between the $5 M_{\odot}$, $Z=0.01$ and the Stromlo model with a small ${ }^{13} \mathrm{C}$ pocket $\left(M_{\text {mix }}=1 \times 10^{-4} M_{\odot}\right)$, while yields of other heavier $s$-process elements are higher in the Stromlo case. Examples include $\mathrm{Zr}$ (factor of 4 higher), and $\mathrm{Ba} / \mathrm{La}$ (roughly a factor of 40 higher), and $\mathrm{Pb}$ (factor of 2.8 higher). That the yields of $\mathrm{Rb}$ are similar is a coincidence: the higher TDU offsets the slower rate for the ${ }^{22} \mathrm{Ne}(\alpha, \mathrm{n}){ }^{25} \mathrm{Mg}$ reaction that we adopt. The MESA calculations adopt the faster NACRE rate for this important neutron producing reaction; Karakas et al. (2012) showed that this rate increases $\mathrm{Rb}$ production. Here we adopt the slower Iliadis et al. (2010) rate. The Stromlo $5 M_{\odot}$ model without a ${ }^{13} \mathrm{C}$ pocket produces fewer heavy elements than the NuGrid model by almost a factor of two in most cases. This indicates two things: the importance of small ${ }^{13} \mathrm{C}$ pockets in intermediate-mass $\mathrm{AGB}$ models and that the NuGrid $5 M_{\odot}$ models have small ${ }^{13} \mathrm{C}$ pockets as a result of the convective boundary mixing scheme employed in the MESA evolutionary calculations (see the discussion in Pignatari et al. 2013). In summary, the NuGrid/ MESA models are qualitatively similar to ours: HBB occurs and produces $\mathrm{N}$, heavy element production occurs and is dominated by the light $s$-process elements around $\mathrm{Rb}$, even in the presence of a small ${ }^{13} \mathrm{C}$ pocket.

\section{CONCLUSIONS}

In this study we presented surface abundances for elements and isotopes, as well as elemental stellar yields for an extensive set of AGB models covering a large range in mass for three metallicities, and including the first nucleosynthesis predictions and yields for twice-solar metallicity AGB models. For solar metallicity and models with a super solar metallicity of $Z=0.03$, we include models from $1 M_{\odot}$ to the $\mathrm{C}-\mathrm{O}$ core limit of $8 M_{\odot}$, noting that the limiting mass at these metallicities will end as a hybrid $\mathrm{CO}(\mathrm{Ne})$ white dwarf. For the lower metallicity models of $Z=0.007$, we include models from $1 M_{\odot}$ to $7.5 M_{\odot}$, where $7 M_{\odot}$ is the $\mathrm{C}-\mathrm{O}$ core limit and the $7.5 M_{\odot}$ is a super-AGB model. This is one of the few predictions of heavy element production for a super-AGB star in the literature.

We include a fine grid of masses which allows us to observe behavior in the surface abundances and yields that would otherwise be missed. An example is the prediction that models between about $4.25 M_{\odot}$ and $4.75 M_{\odot}$ with solar metallicity that experience $\mathrm{HBB}$ have low ${ }^{12} \mathrm{C} /{ }^{13} \mathrm{C}$ ratios $<10$ but become $\mathrm{C}$-rich. A fine grid of masses is required in order to provide accurate yield predictions for Galactic chemical evolution models, as discussed by Izzard et al. (2004) in the context of synthetic, rapid AGB models.

We find that the surface abundances and yields of the super solar metallicity AGB stars of $Z=0.03$ are different to their lower metallicity counterparts. Only models above $2.5 M_{\odot}$ experience TDU and only models above $5 M_{\odot}$ experience HBB. We find a narrow range of carbon star production, between $2.5 M_{\odot}$ and $4 M_{\odot}$, even when we include convective overshoot. Without overshoot, the range decreases to $3.25-4 M_{\odot}$, as discussed in Karakas (2014). This mass range also dictates which masses produce $s$-process elements, as shown in Sections 3 and 4. Super solar metallicity models of intermediate-mass are predicted to produce only light elements from HBB and no heavy elements. Interestingly, these models are all net Li producers, including the most massive models of $8 M_{\odot}$ near the CO-core mass limit which experience efficient HBB. This is in contrast to models of lower metallicity and solar composition, which only have positive net lithium production near the minimum stellar mass for $\mathrm{HBB} \approx 4.5 M_{\odot}$. Higher mass models destroy Li by the end of the AGB phase.

We weight the yields by a Salpeter IMF to show how the yields and $s$-process abundances change in a stellar population; this gives an indication how important each mass range is for chemical evolution. As found elsewhere (Travaglio et al. 2004; Bisterzo et al. 2014), the yields from the intermediate-mass AGB stars do not play an important role at these metallicities, except for $\mathrm{N}$ and $\mathrm{Rb}$. Production of a substantial yield of $\mathrm{Rb}$ from AGB stars requires the contribution from stars of $\approx 4 M_{\odot}$ that include both the ${ }^{13} \mathrm{C}$ and ${ }^{22} \mathrm{Ne}$ neutron sources. Models with the ${ }^{22} \mathrm{Ne}$ source alone are not predicted to play a major role in the production of $\mathrm{Rb}$ or other heavy elements in the Galaxy, at least at these metallicities.

Finally, we compare our models to the FRUITY nucleosynthesis predictions from Cristallo et al. (2015) and the NuGrid AGB models and yields from Pignatari et al. (2013). We find reasonable qualitative agreement between the FRUITY models of low-mass AGB stars of $1.5 M_{\odot}$ and $3 M_{\odot}$ for all metallicities (except $Z=0.03$, for which no FRUITY models are available to compare). However, we find large discrepancies between the FRUITY $6 M_{\odot}$ intermediate-mass models and ours, while our $5 M_{\odot}$ model is qualitatively similar to the $5 M_{\odot}$ NuGrid models. While this is a problem that may be resolved through a careful comparison with observations, the yields of these intermediate-mass AGB stars are not important for bulk Galactic chemical evolution studies. There may be places in the Galaxy, however, where the yields of intermediate-mass AGB stars are important and these possibly include Galactic globular clusters (Ventura et al. 2013; Shingles et al. 2014; Straniero et al. 2014). One issue with models of intermediate-mass AGB stars is the paucity of observations, which are required to confront theoretical predictions. Future observations of bright intermediate-mass AGB stars in stellar populations of varying 
metallicity would be an invaluable resource in furthering our understanding of these enigmatic objects.

The authors thank the referee for encouraging us to dig deeper into the differences between our models and other published results. We alsothank Marco Pignatari for providing information about the NuGrid AGB models and yields and Sergio Cristallo for providing detailed information about the FRUITY models. This research was undertaken with the assistance of resources from the National Computational Infrastructure (NCI), which is supported by the Australian Government. A.I.K. was supported through an Australian Research Council Future Fellowship (FT110100475). M.L. is a Momentum ("Lendület-2014" Programme) project leader of the Hungarian Academy of Sciences.

\section{APPENDIX \\ APPENDIX MATERIAL}

Examples of each of the data table types are included in the appendix.

\section{REFERENCES}

Abia, C., Domínguez, I., Gallino, R., et al. 2002, ApJ, 579, 817

Abia, C., \& Isern, J. 1997, MNRAS, 289, L11

Akram, W., Schönbächler, M., Bisterzo, S., \& Gallino, R. 2015, GeCoA, 165,484

Akram, W., Schönbächler, M., Sprung, P., \& Vogel, N. 2013, ApJ, 777, 169

Amari, S., Nittler, L. R., Zinner, E., et al. 2001a, ApJ, 546, 248

Amari, S., Nittler, L. R., Zinner, E., Lodders, K., \& Lewis, R. S. 2001b, ApJ, 559,463

Angulo, C., Arnould, M., Rayet, M., et al. 1999, NuPhA, 656, 3

Asplund, M., Grevesse, N., Sauval, A. J., \& Scott, P. 2009, ARA\&A, 47, 481

Bisterzo, S., Gallino, R., Straniero, O., Cristallo, S., \& Käppeler, F. 2010, MNRAS, 404, 1529

Bisterzo, S., Gallino, R., Straniero, O., Cristallo, S., \& Käppeler, F. 2011, MNRAS, 418, 284

Bisterzo, S., Travaglio, C., Gallino, R., Wiescher, M., \& Käppeler, F. 2014, ApJ, 787, 10

Blöcker, T. 1995, A\&A, 297, 727

Bonačić Marinović, A., Lugaro, M., Reyniers, M., \& van Winckel, H. 2007, A\&A, 472, L1

Boothroyd, A. I., \& Sackmann, I.-J. 1999, ApJ, 510, 232

Burkhardt, C., \& Schönbächler, M. 2015, GeCoA, 165, 361

Busso, M., Gallino, R., Lambert, D. L., Travaglio, C., \& Smith, V. V. 2001, ApJ, 557, 802

Busso, M., Gallino, R., \& Wasserburg, G. J. 1999, ARA\&A, 37, 239

Busso, M., Palmerini, S., Maiorca, E., et al. 2010, ApJL, 717, L47

Charbonnel, C. 1994, A\&A, 282, 811

Charbonnel, C., \& Zahn, J.-P. 2007, A\&A, 467, L15

Cristallo, S., Piersanti, L., Straniero, O., et al. 2011, ApJS, 197, 17

Cristallo, S., Straniero, O., Gallino, R., et al. 2009, ApJ, 696, 797

Cristallo, S., Straniero, O., Piersanti, L., \& Gobrecht, D. 2015, ApJS, 219, 40

Dauphas, N., Marty, B., \& Reisberg, L. 2002, ApJL, 569, L139

Decin, L., Justtanont, K., De Beck, E., et al. 2010, A\&A, 521, L4

De Smedt, K., Van Winckel, H., Karakas, A. I., et al. 2012, A\&A, 541, A67

Doherty, C. L., Gil-Pons, P., Lau, H. H. B., Lattanzio, J. C., \& Siess, L. 2014 MNRAS, 437, 195

Doherty, C. L., Gil-Pons, P., Siess, L., Lattanzio, J. C., \& Lau, H. H. B. 2015 , MNRAS, 446, 2599

Eggleton, P. P., Dearborn, D. S. P., \& Lattanzio, J. C. 2008, ApJ, 677, 581 Fishlock, C. K., Karakas, A. I., Lugaro, M., \& Yong, D. 2014, ApJ, 797, 44 Fonfría, J. P., Cernicharo, J., Richter, M. J., et al. 2015, MNRAS, 453, 439 Freeman, K., \& Bland-Hawthorn, J. 2002, ARA\&A, 40, 487

Frost, C. A., \& Lattanzio, J. C. 1996, ApJ, 473, 383

Gallino, R., Arlandini, C., Busso, M., et al. 1998, ApJ, 497, 388

García-Hernández, D. A., García-Lario, P., Plez, B., et al. 2006, Sci, 314, 1751

García-Hernández, D. A., Manchado, A., Lambert, D. L., et al. 2009, ApJL, 705, L31

García-Hernández, D. A., Zamora, O., Yagüe, A., et al. 2013, A\&A, 555, L3
Goriely, S., \& Siess, L. 2004, A\&A, 421, L25

Groenewegen, M. A. T., van den Hoek, L. B., \& de Jong, T. 1995, A\&A, 293, 381

Halabi, G. M., \& Eid, M. E. 2015, MNRAS, 451, 2957

Harris, M. J., \& Lambert, D. L. 1984, ApJ, 285, 674

Harris, M. J., Lambert, D. L., Hinkle, K. H., Gustafsson, B., \& Eriksson, K. 1987, ApJ, 316, 294

Harris, M. J., Lambert, D. L., \& Smith, V. V. 1985a, ApJ, 292, 620

Harris, M. J., Lambert, D. L., \& Smith, V. V. 1985b, ApJ, 299, 375

Hedrosa, R. P., Abia, C., Busso, M., et al. 2013, ApJL, 768, L11

Herwig, F. 2004, ApJ, 605, 425

Herwig, F. 2005, ARA\&A, 43, 435

Herwig, F., Langer, N., \& Lugaro, M. 2003, ApJ, 593, 1056

Hoppe, P., Annen, P., Strebel, R., et al. 1997, ApJL, 487, L101

Iliadis, C., Longland, R., Champagne, A. E., Coc, A., \& Fitzgerald, R. 2010, $\mathrm{NuPhA}, 841,31$

Izzard, R. G., Tout, C. A., Karakas, A. I., \& Pols, O. R. 2004, MNRAS, 350,407

Justtanont, K., Barlow, M. J., Blommaert, J., et al. 2015, A\&A, 578, A115

Justtanont, K., Teyssier, D., Barlow, M. J., et al. 2013, A\&A, 556, A101

Kahane, C., Cernicharo, J., Gomez-Gonzalez, J., \& Guelin, M. 1992, A\&A, 256, 235

Kahane, C., Dufour, E., Busso, M., et al. 2000, A\&A, 357, 669

Kalirai, J. S., Marigo, P., \& Tremblay, P.-E. 2014, ApJ, 782, 17

Kamath, D., Karakas, A. I., \& Wood, P. R. 2012, ApJ, 746, 20

Karakas, A. I. 2010, MNRAS, 403, 1413

Karakas, A. I. 2014, MNRAS, 445, 347

Karakas, A. I., Campbell, S. W., \& Stancliffe, R. J. 2010, ApJ, 713, 374

Karakas, A. I., García-Hernández, D. A., \& Lugaro, M. 2012, ApJ, 751, 8

Karakas, A. I., \& Lattanzio, J. C. 2003, PASA, 20, 279

Karakas, A. I., \& Lattanzio, J. C. 2014, PASA, 31, 30

Karakas, A. I., Lattanzio, J. C., \& Pols, O. R. 2002, PASA, 19, 515

Karakas, A. I., Lugaro, M., \& Gallino, R. 2007, ApJL, 656, L73

Karakas, A. I., Lugaro, M., Wiescher, M., Goerres, J., \& Ugalde, C. 2006, ApJ, 643,471

Karakas, A. I., Marino, A. F., \& Nataf, D. M. 2014, ApJ, 784, 32

Karakas, A. I., van Raai, M. A., Lugaro, M., Sterling, N. C., \& Dinerstein, H. L. 2009, ApJ, 690, 1130

Khouri, T., de Koter, A., Decin, L., et al. 2014, A\&A, 570, A67

Kobayashi, C., Izutani, N., Karakas, A. I., et al. 2011a, ApJL, 739, L57

Kobayashi, C., Karakas, A. I., \& Umeda, H. 2011b, MNRAS, 414, 3231

Lagarde, N., Charbonnel, C., Decressin, T., \& Hagelberg, J. 2011, A\&A, 536, A28

Lattanzio, J. C. 1986, ApJ, 311, 708

Lattanzio, J. C., Frost, C. A., Cannon, R. C., \& Wood, P. R. 1997, NuPhA, 621,435

Lattanzio, J. C., Siess, L., Church, R. P., et al. 2015, MNRAS, 446, 2673

Lebzelter, T., Lederer, M. T., Cristallo, S., et al. 2008, A\&A, 486, 511

Lebzelter, T., Straniero, O., Hinkle, K. H., Nowotny, W., \& Aringer, B. 2015, A\&A, 578, A33

Lederer, M. T., \& Aringer, B. 2009, A\&A, 494, 403

Lugaro, M., Campbell, S. W., Van Winckel, H., et al. 2015, A\&A, 583, A77

Lugaro, M., Davis, A. M., Gallino, R., et al. 2003, ApJ, 593, 486

Lugaro, M., Heger, A., Osrin, D., et al. 2014a, Sci, 345, 650

Lugaro, M., Karakas, A. I., Stancliffe, R. J., \& Rijs, C. 2012, ApJ, 747, 2

Lugaro, M., Tagliente, G., Karakas, A. I., et al. 2014b, ApJ, 780, 95

Lugaro, M., Ugalde, C., Karakas, A. I., et al. 2004, ApJ, 615, 934

Marigo, P., Bressan, A., Nanni, A., Girardi, L., \& Pumo, M. L. 2013, MNRAS, 434, 488

Marigo, P., Girardi, L., \& Bressan, A. 1999, A\&A, 344, 123

Milam, S. N., Woolf, N. J., \& Ziurys, L. M. 2009, ApJ, 690, 837

Miller Bertolami, M. M. 2015, A\&A, 588, A25

Mollá, M., Cavichia, O., Gavilán, M., \& Gibson, B. K. 2015, MNRAS, 451, 3693

Nollett, K. M., Busso, M., \& Wasserburg, G. J. 2003, ApJ, 582, 1036

Nomoto, K., Kobayashi, C., \& Tominaga, N. 2013, ARA\&A, 51, 457

Palmerini, S., La Cognata, M., Cristallo, S., \& Busso, M. 2011, ApJ, 729, 3

Peng, T.-C., Humphreys, E. M. L., Testi, L., et al. 2013, A\&A, 559, L8

Piersanti, L., Cristallo, S., \& Straniero, O. 2013, ApJ, 774, 98

Pignatari, M., Herwig, F., Hirschi, R., et al. 2013, ApJS, in press

Prantzos, N. 2012, A\&A, 542, A67

Romano, D., Karakas, A. I., Tosi, M., \& Matteucci, F. 2010, A\&A, 522, A32 Shingles, L. J., Doherty, C. L., Karakas, A. I., et al. 2015, MNRAS, 452, 2804

Shingles, L. J., \& Karakas, A. I. 2013, MNRAS, 431, 2861

Shingles, L. J., Karakas, A. I., Hirschi, R., et al. 2014, ApJ, 795, 34

Siess, L. 2006, A\&A, 448, 717 
Siess, L., Goriely, S., \& Langer, N. 2004, A\&A, 415, 1089

Smith, V. V., \& Lambert, D. L. 1989, ApJL, 345, L75

Smith, V. V., \& Lambert, D. L. 1990, ApJL, 361, L69

Stancliffe, R. J. 2010, MNRAS, 403, 505

Stancliffe, R. J., Izzard, R. G., \& Tout, C. A. 2005, MNRAS, 356, L1

Stancliffe, R. J., \& Jeffery, C. S. 2007, MNRAS, 375, 1280

Straniero, O., Cristallo, S., \& Piersanti, L. 2014, ApJ, 785, 77

Straniero, O., Gallino, R., \& Cristallo, S. 2006, NuPhA, 777, 311

Tinsley, B. M. 1980, FcPh, 5, 287

Travaglio, C., Gallino, R., Arnone, E., et al. 2004, ApJ, 601, 864

Travaglio, C., Randich, S., Galli, D., et al. 2001, ApJ, 559, 909

van Raai, M. A., Lugaro, M., Karakas, A. I., García-Hernández, D. A., \& Yong, D. 2012, A\&A, 540, A44

van Raai, M. A., Lugaro, M., Karakas, A. I., \& Iliadis, C. 2008, A\&A, 478, 521
Vassiliadis, E., \& Wood, P. R. 1993, ApJ, 413, 641

Ventura, P., Carini, R., \& D’Antona, F. 2011, MNRAS, 415, 3865

Ventura, P., \& D'Antona, F. 2005a, A\&A, 431, 279

Ventura, P., \& D'Antona, F. 2005b, A\&A, 439, 1075

Ventura, P., D’Antona, F., \& Mazzitelli, I. 2000, A\&A, 363, 605

Ventura, P., Di Criscienzo, M., Carini, R., \& D’Antona, F. 2013, MNRAS, 431, 3642

Weiss, A., \& Ferguson, J. W. 2009, A\&A, 508, 1343

Whitelock, P. A., Feast, M. W., \& van Leeuwen, F. 2008, MNRAS, 386, 313

Wood, P. R., Bessell, M. S., \& Fox, M. W. 1983, ApJ, 272, 99

Zamora, O., García-Hernández, D. A., Plez, B., \& Manchado, A. 2014, A\&A, $564, \mathrm{~L} 4$

Zinner, E. 2014, in Meteorites and Cosmochemical Processes, Treatise on Geochemistry, Vol. 1, ed. A. M. Davis (2nd ed.; New York: Elsevier) 